\title{
Optimal and Suboptimal Resource Allocation in MIMO Cooperative Cognitive Radio Networks
}

\author{
Mehdi Ghamari Adian' and Mahin Ghamari Adyan² \\ ${ }^{1}$ Electrical Engineering Department, University of Zanjan, Zanjan 45371-38791, Iran \\ ${ }^{2}$ Electrical Engineering Department, University of Tehran, Tehran 24785-54789, Iran \\ Correspondence should be addressed to Mehdi Ghamari Adian; mehdi.ghamari@aut.ac.ir
}

Received 11 April 2014; Revised 19 July 2014; Accepted 21 July 2014; Published 31 August 2014

Academic Editor: Kai-Kit Wong

Copyright (C) 2014 M. Ghamari Adian and M. Ghamari Adyan. This is an open access article distributed under the Creative Commons Attribution License, which permits unrestricted use, distribution, and reproduction in any medium, provided the original work is properly cited.

\begin{abstract}
The core aim of this work is the maximization of the achievable data rate of the secondary user pairs (SU pairs), while ensuring the QoS of primary users (PUs). All users are assumed to be equipped with multiple antennas. It is assumed that when PUs are present, the direct communication between SU pairs introduces intolerable interference to PUs and thereby SUs transmit signal using the cooperation of one of the SUs and avoid transmission in the direct channel. In brief, an adaptive cooperative strategy for MIMO cognitive radio networks is proposed. At the presence of PUs, the issue of joint relay selection and power allocation in underlay MIMO cooperative cognitive radio networks (U-MIMO-CCRN) is addressed. The optimal approach for determining the power allocation and the cooperating SU is proposed. Besides, the outage probability of the proposed system is further derived. Due to high complexity of the optimal approach, a low complexity approach is further proposed and its performance is evaluated using simulations. The simulation results reveal that the performance loss due to the low complexity approach is only about $14 \%$, while the complexity is greatly reduced.
\end{abstract}

\section{Introduction}

Since the issuance of the report of Federal Communications Commission (FCC) in 2002, which revealed the spectrum inefficiency in the incumbent wireless communication systems, cognitive radio (CR) has been regarded as one potential technology to activate the utilization of spectrum resources in the recent evolution of wireless communication systems [1]. As a consequence, the overlay and underlay modes can be developed, based on the definitions of spectrum holes in [1] and the operation modes in [2], to use the white and gray spectrum holes, respectively.

Cognitive radio (CR), MIMO communications, and cooperative communications are among the most promising solutions to improve spectrum utilization and efficiency. Dynamic and opportunistic spectrum access allows CR nodes to communicate on temporarily idle or underutilized frequencies. MIMO systems boost spectral efficiency by having a multiantenna node that simultaneously transmit multiple data streams. To further enhance the performance of cognitive radio networks, a cooperative relay network can be incorporated. Thus, in the underlay CR system with an interference temperature (IT) limit, the cooperative relay networks can also be applied to have a better capacity and error rate performance, trade-off between achievable rate and network lifetime, maximum signal-to-interference-plusnoise ratio (SINR) at the destination node, better channel utilization by multihop relay, maximum throughput, and reduced interference via beamforming and maximum SINR using cooperative beamforming. A timely issue is to embrace recent innovations of the three technologies into a single system.

Joint problems of relay selection and resource allocation in CR networks (CRNs) have attracted extensive research interests due to its more effective spectrum utilization [38]. The authors in [3] consider a cooperative cognitive radio network (CCRN) in which the relays are selected among the existing SUs. Moreover, the QoS of the relays 
should be ensured. For CCRN with decode-and-forward strategy, two relay selection schemes, namely, a full-channel state information (CSI) based best relay selection (BRS) and a partial CSI based best relay selection (PBRS), were proposed in [4]. In order to obtain an optimal subcarrier pairing, relay assignment and power allocation in MIMOOFDM based CCRN, the dual decomposition technique was recruited in [5] to maximize the sum rate subject to the interference temperature limit of the PUs. Moreover, due to high computational complexity of the optimal approach, a suboptimal algorithm was further proposed in [5]. The issue of joint relay selection and power allocation in twoway CCRN was considered in [6]. A suboptimal approach for reducing the complexity of joint relay selection and power allocation in CCRN was proposed in [7]. The network coding opportunities existing in cooperative communications that can further increase the capacity was exploited in [8]. Furthermore, the reformulation and linearization techniques to the original optimization problems with nonlinear and nonconvex objective functions were applied such that the proposed algorithms can produce high competitive solutions in a timely manner.

The issue of resource allocation in MIMO CRNs was explored in [9-14]. The authors in [9] presented a low complexity semidistributed algorithm for resource allocation in MIMO-OFDM based CR networks, using game theory approach, the strong duality in convex optimization, and the primal decomposition method. In [11], the authors extended the pricing concept to MIMO-OFDM based CR networks and presented two iterative algorithms for resource allocation in such systems. In order to obtain an optimal subcarrier pairing, relay assignment, and power allocation in MIMOOFDM based CCRN, the dual decomposition technique was recruited in [12] to maximize the sum rate subject to the interference temperature limit of the PUs. Moreover, because of high computational complexity of the optimal approach, a suboptimal algorithm was further proposed in [12, 13]. An approach for resource allocation based on beamforming with reduced complexity in MIMO cooperative cognitive radio networks was presented in [14], where a suboptimal approach with reduced complexity was further proposed to jointly determine the transmit beamforming (TB) and cooperative beamforming $(\mathrm{CB})$ weight vectors along with antenna subset selection in MIMO-CCRN. The problem of resource allocation in a spectrum leasing scenario in cooperative cognitive radio networks was addressed in [10], where the SU pair allocates the whole of its transmission power in a portion of transmission frame to relay the primary signals. In return, the PU pairs lease their unused portion of transmission frame to the SU pair.

The optimal resource allocation in MIMO cognitive radio networks with heterogeneous secondary users and centralized and distributed users was investigated in [15]. The authors in [16] modeled the problem of joint relay selection and power allocation in MIMO-OFDM based CCRN as a two-level cooperative game problem with two objectives. The first objective is to assign each weak SU to one of the relays (rich SUs) through solving a problem achieved by a nontransferable utility coalition graph game and the second one is to jointly allocate available channels to the SUs such that no subchannel is allocated to more than one SU and simultaneously optimize the transmit covariance matrices of nodes based on the Nash bargaining solution, which is the second level of the game.

In this paper, we consider the opportunistic spectrum access in MIMO cognitive radio networks (MIMO-CRN) to ensure the SUs' continuous transmission and reduce its outage probability without interfering the PUs. The desired link is considered as the MIMO link between two SUs, SU transmitter (SU TX), and SU receiver (SU RX). All the users are assumed to be equipped with multiple antennas. We recruit spectrum sensing technology to detect the presence of the PUs. If the PUs are absent, the SU TX communicates the SU RX straightly. Otherwise, the transmit power of SU TX has to be reduced and SU TX transmits to SU RX using the cooperation of one the existing SUs. The cooperating SU is determined using the best relay selection algorithm. To be more accurate, when a PU transmits signal in the system, the joint problems of opportunistic relay selection and power allocation in the context of MIMO CRN to maximize the end-to-end achievable data rate of MIMO CRN need to be considered. Our focus is on the amplify-andforward (AF) relay strategy. An obvious reason is that AF has low complexity since no decoding/encoding is needed. This benefit is even more attractive in MIMO-CRN, where decoding multiple data streams could be computationally intensive. Moreover, a more important reason is that AF outperforms decode-and-forward (DF) strategy in terms of network capacity scaling: in general, as the number of relays increases in MIMO-CRN, the effective signal-to-noise ratio (SNR) under AF scales linearly, as opposed to being a constant under DF [17].

To the best of our knowledge, the joint problems of relay selection and power allocation in MIMO cognitive radio networks has not been explored yet. The main contributions of the paper are as follows.

(i) The optimal structure of amplification matrix at the cooperating SU and transmit covariance matrix at the transmitter are determined at the presence of PUs.

(ii) The optimal approach for solving the problem based on the dual method is presented and then a low complexity suboptimal approach is proposed to solve the joint problems of relay selection and power allocation in underlay MIMO CRN.

(iii) The outage performance of the desired SU link is analyzed.

The remainder of this paper is organized as follows. Section 2 presents the system model and general formulation of the problem. In Section 3, the structure of optimal power allocation matrices is studied. Based on these structural results, we simplify and reformulate the optimization problem. The optimization algorithms, including the optimal and suboptimal approach, are discussed in Section 4. In Section 5, the outage probability of the desired link is analyzed. Numerical results are provided in Section 6 and Section 7 concludes this paper. 
Notation. Uppercase and lowercase boldface denote matrices and vectors, respectively. The operators $(\cdot)^{H},|\cdot|, \operatorname{Tr}(\cdot)$, and $(\cdot)^{+}$ are Hermitian (complex conjugate), determinant, trace, and pseudoinverse operators, respectively.

\section{System Model}

We consider a scenario where a secondary network, consisting of $N_{\mathrm{SU}}+2$ SUs coexists with a primary network, consisting of $N_{\mathrm{PU}} \mathrm{PU}$ pair. In this paper, the communication between two SUs is considered, which is also referred to as the desired SU link. The SU transmitter (SU TX) transmits signals to SU receiver (SU RX) either in the direct link or taking advantage of the cooperation of one of the SUs, depending on the presence of the PUs. When the PUs are absent, the SU TX simply communicates the SU RX directly. However, in order not to induce intolerable interference on the PUs, transmission from SU TX to SU RX at the presence of PUs takes place in two consecutive time-slots using the cooperation of one the existing SU.

2.1. The Transmission Process at the Presence of PUs. When PU pairs are present, the direct communications between the SU TX and SU RX may impose intolerable interference on the PUs. The cooperation of one of SUs with the desired SU link can provide the possibility of reducing the transmit power of the SUs and thereby less interference is imposed on the PU pairs. This is shown in Figure 1. The SU TX is equipped with cognitive radio capabilities and senses the available spectrum bands precisely.

The selected cooperating SU cannot transmit and receive in the same channel at the same time, due to self-interference. Thus, a transmission from SU TX to SU RX at the presence of PUs takes two time-slots. This is also depicted in Figure 1. In the first time-slot, the SU TX transmits signals to all the existing SUs in the CR network. In the second time-slot, one of the SUs is selected to cooperate with the SU TX by amplifying its received signal and forwarding it to the SU RX. All the transmissions in the SU system need to be regulated in order to avoid excessive interference on the PU pair. The set of candidate SUs to cooperate with the desired SU link is denoted by $\mathcal{S}_{R}$. Besides, the set of PU pairs is also denoted by $\mathcal{S}_{\mathrm{PU}}$. It is further assumed that all the users, including the SUs and the Pus, are equipped with multiple antennas. Without loss of generality and for ease of exposition, we assume that all the candidate SUs to cooperate with desired link are equipped with $N_{r}$ antennas and the PUs with $N_{p}$ antennas. The number of antennas at SU TX and SU RX is also $N_{s}$ and $N_{d}$, respectively. $\mathbf{H}_{s r, i} \in \mathbb{C}^{N_{r} \times N_{s}}$ represents the channel matrix form SU TX to SU $i$ and $\mathbf{H}_{r d, i} \in \mathbb{C}^{N_{r} \times N_{d}}$ represents the channel form SU $i$ to SU RX. All the channels are modeled as Rayleigh fading channels and they are invariant during one time-slot.

Remark 1. In this paper, we assume that a central controller is available, so that the network channel state information and sensing results can be reliably gathered for centralized processing. Notice that the centralized CRNs are valid in

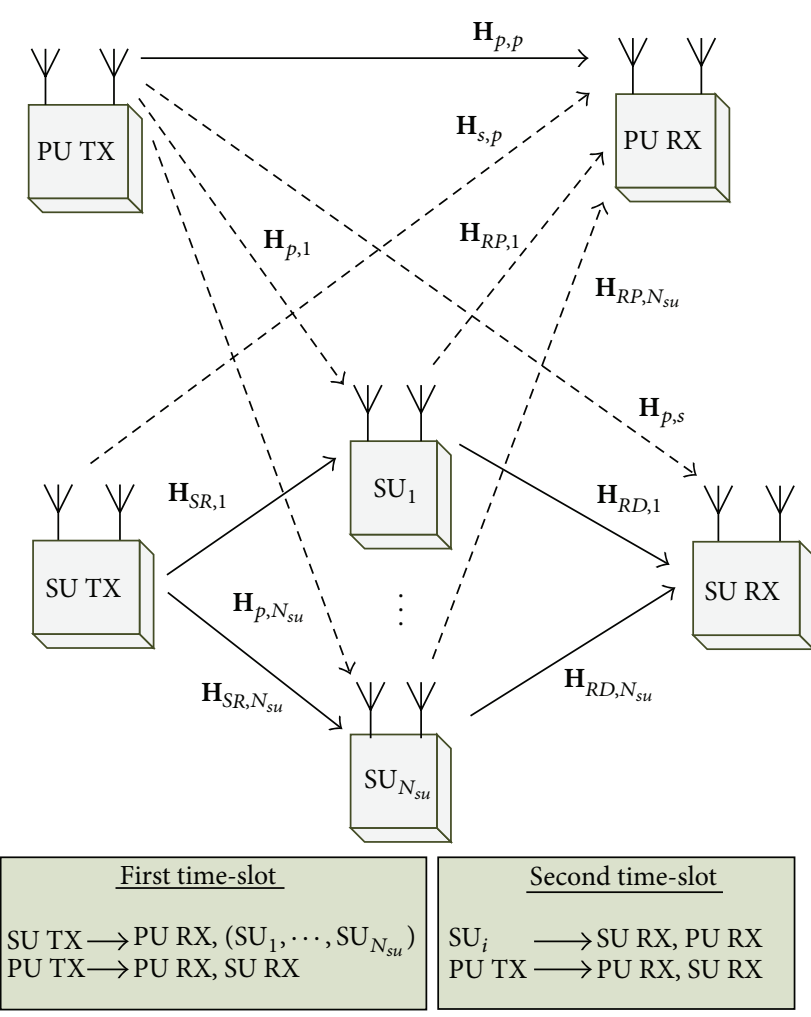

$\longrightarrow$ Information channel

$-\rightarrow$ Interference channel

FIGURE 1: System model with the two transmission hops, the desired SU link, and other secondary and primary users.

IEEE 802.22 standard [18], where the cognitive systems operate on a cellular basis and the central controller can be embedded with a base station (BS). This assumption is also reasonable if a spectrum broker exists in CRNs for managing spectrum leasing and access $[19,20]$. Such centralized approach is commonly used in a variety of CRNs (e.g., [19-24]). Compared with distributed approaches, a CCRN having a central manager that possesses detailed information about the wireless network enables highly efficient network configuration and better enforcement of a complex set of policies [20].

2.2. Problem Formulation. The received signal at $i$ th $\mathrm{SU}$ can be written as

$$
\mathbf{y}_{r, i}=\mathbf{H}_{s r, i} \mathbf{x}_{s, i}+\mathbf{n}_{r, i}, \quad \forall i \in \mathcal{S}_{R}
$$

where the transmit signal of SU TX, intended for $\mathrm{SU} i$, is denoted by $\mathbf{x}_{s, i} \in \mathbb{C}^{N_{s} \times 1} ; \mathbf{n}_{r, i} \in \mathbb{C}^{N_{r} \times 1}$ is a Gaussian vector. The entries of $\mathbf{n}_{r, i}$ are assumed to be independent and identically distributed random variables, having zero means and variances $\sigma_{r}^{2}$; that is, $n_{r, i} \sim \mathscr{C} \mathscr{N}\left(0, \sigma_{r}^{2} \mathbf{I}_{N_{r}}\right)$. To provide more clarifications, in order to take the effect from the PU transmission into consideration and similar to $[25,26]$, the distribution of $\mathbf{n}_{r, i}$ is $\mathscr{C} \mathcal{N}\left(0,\left(\sigma_{0}^{2}+\sum_{n \in \mathcal{S}_{\mathrm{PU}}} \operatorname{Tr}\left(\mathbf{H}_{p, i, n} \mathbf{Q}_{p, n} \mathbf{H}_{p, i, n}^{H}\right)\right) \mathbf{I}_{N_{r}}\right)$, where $\mathbf{H}_{P, i, n}$ represents the channel matrix from the $n$th PU to the 
$i$ th SU; $\mathbf{Q}_{P, n}$ denotes the transmit covariance matrix of the $n$th PU; $\sigma_{0}^{2}$ is the noise power and we assume that all the links have the same amount of noise power. Therefore, $\sigma_{r, i}^{2}=\sigma_{0}^{2}+$ $\sum_{n \in \mathcal{S}_{\mathrm{PU}}} \operatorname{Tr}\left(\mathbf{H}_{p, i, n} \mathbf{Q}_{p, n} \mathbf{H}_{p, i, n}^{H}\right)$ denotes the power of noise and interference in the link from SU TX to the $i$ th SU. Without losing the generality and for ease of exposition, we further assume that $\sigma_{r, i}^{2}=\sigma_{r}^{2}$, for all $i \in \mathcal{S}_{R}$. Suppose that SU $i$ is selected to cooperate with the desired SU link. Then, the received signal at the $S U R X$ (destination) from $S U i$ is given by

$$
\begin{aligned}
\mathbf{y}_{d} & =\mathbf{H}_{r d, i} \mathbf{A}_{i} \mathbf{y}_{r, i}+\mathbf{n}_{d} \\
& =\mathbf{H}_{r d, i} \mathbf{A}_{i} \mathbf{H}_{s r, i} \mathbf{x}_{s, i}+\mathbf{H}_{r d, i} \mathbf{A}_{i} \mathbf{n}_{r, i}+\mathbf{n}_{d},
\end{aligned}
$$

where $\mathbf{A}_{i}$ represents the amplification matrix, used at $\mathrm{SU} i$; similar to $\mathbf{n}_{r, i}, \mathbf{n}_{d} \in \mathbb{C}^{N_{d} \times 1}$ is also a Gaussian vector and $\sigma_{r, i}^{2}=\sigma_{0}^{2}+\sum_{n \in \mathcal{S}_{\mathrm{PU}}} \operatorname{Tr}\left(\mathbf{H}_{p, s, n} \mathbf{Q}_{p, n} \mathbf{H}_{p, s, n}^{H}\right)$, where $\mathbf{H}_{p, s, n}$ denotes the channel matrix from the $n$th PU to SU RX. As a result of cooperation of one of the SUs, for example, SU $i$, the achievable data rate in the desired link can be written as

$$
\begin{aligned}
R_{i}=\frac{1}{2} \log _{2} \mid \mathbf{I}_{N_{d}} & +\mathbf{H}_{r d, i} \mathbf{A}_{i} \mathbf{H}_{s r, i} \mathbf{Q}_{i} \mathbf{H}_{s r, i}^{H} \mathbf{A}_{i}^{H} \mathbf{H}_{r d, i}^{H} \\
& \times\left(\sigma_{d}^{2} \mathbf{I}_{N_{d}}+\sigma_{r}^{2} \mathbf{H}_{r d, i} \mathbf{A}_{i} \mathbf{A}_{i}^{H} \mathbf{H}_{r d, i}^{H}\right)^{-1} \mid,
\end{aligned}
$$

where $\mathbf{Q}_{i}=E\left\{\mathbf{x}_{s, i} \mathbf{x}_{s, i}^{H}\right\}$ denotes the transmit covariance matrix of SU TX, intended for SU $i$. The transmit power of SU TX to each SU is restricted to $P_{T}$; that is, $\operatorname{Tr}\left(\mathbf{Q}_{i}\right) \leq P_{T}$. Furthermore, the maximum transmit power of the SU $i$, if selected as the cooperative relay, is $P_{R}$; that is, $\operatorname{Tr}\left[\mathbf{A}_{i}\left(\sigma_{r}^{2} \mathrm{I}_{N_{r}}+\right.\right.$ $\left.\left.\mathbf{H}_{s r, i} \mathbf{Q}_{i} \mathbf{H}_{s r, i}^{H}\right) \mathbf{A}_{i}^{H}\right] \leq P_{R}$. The coefficient $1 / 2$ in (3) is due to the fact that cooperative transmission only uses half of resources (e.g., time-slots, frequency bands, etc.). The PUs must not be disturbed as a result of transmission by SU TX and further the cooperation of the selected SU with the SU TX. In this way, the interference power constraints on the PUs are provided by $\operatorname{Tr}\left(\mathbf{H}_{s, p, n} \mathbf{Q}_{i} \mathbf{H}_{s, p, n}^{H}\right) \leq P_{I, 1}$ and $\operatorname{Tr}\left\{\mathbf{H}_{i, p, n}\left[\mathbf{A}_{i}\left(\sigma_{r}^{2} \mathbf{I}_{N_{r}}+\mathbf{H}_{s r, i} \mathbf{Q}_{i} \mathbf{H}_{s r, i}^{H}\right) \mathbf{A}_{i}^{H}\right] \mathbf{H}_{i, p, n}^{H}\right\} \leq P_{I, 2}$, for all $n \in \mathcal{S}_{\mathrm{PU}}$, where the $\mathrm{SU} i$ is selected to cooperate with the SU TX. Moreover, $\mathbf{H}_{i, p, n}$ and $\mathbf{H}_{s, p, n}$ represent the channel from SU $i$ and SU TX to $n$th PU RX, respectively. Evidently, the maximum tolerable interference at the PUs is $P_{I, 1}+P_{I, 2}$. One of the aims of this work is to optimally select the cooperating SU and also calculate the optimum power allocation (finding the optimal $\mathbf{Q}_{i}$ and $\mathbf{A}_{i}$ ) in the proposed system, which can be formulated as

$$
\begin{aligned}
\left(\mathbf{Q}_{i}^{*}, \mathbf{A}_{i}^{*}\right) & =\underset{\mathbf{Q}, \mathbf{A}_{i}}{\arg \max } R_{i}\left(\mathbf{Q}_{i}, \mathbf{A}_{i}\right), \\
i & =\underset{i}{\arg \max } R_{i}\left(\mathbf{Q}_{i}^{*}, \mathbf{A}_{i}^{*}\right),
\end{aligned}
$$

s.t. $\operatorname{Tr}\left(\mathbf{Q}_{i}\right) \leq P_{T}$

$$
\begin{aligned}
& \operatorname{Tr}\left[\mathbf{A}_{i}\left(\sigma_{r}^{2} \mathbf{I}_{N_{r}}+\mathbf{H}_{s r, i} \mathbf{Q}_{i} \mathbf{H}_{s r, i}^{H}\right) \mathbf{A}_{i}^{H}\right] \leq P_{R}, \\
& \operatorname{Tr}\left(\mathbf{H}_{s, p, n} \mathbf{Q}_{i} \mathbf{H}_{s, p, n}^{H}\right) \leq P_{I, 1},
\end{aligned}
$$

$$
\begin{array}{r}
\operatorname{Tr}\left\{\mathbf{H}_{i, p, n}\left[\mathbf{A}_{i}\left(\sigma_{r}^{2} \mathbf{I}_{N_{r}}+\mathbf{H}_{s r, i} \mathbf{Q}_{i} \mathbf{H}_{s r, i}^{H}\right) \mathbf{A}_{i}^{H}\right] \mathbf{H}_{i, p, n}^{H}\right\} \leq P_{I, 2} \\
\mathbf{A}_{i} \geq 0, \quad \mathbf{Q}_{i} \geq 0
\end{array}
$$

for all $n \in \mathcal{S}_{\mathrm{PU}}$, where $\mathbf{Q}_{i}^{*}$ and $\mathbf{A}_{i}^{*}$ are the optimum transmit covariance and amplification matrices. For convenience, we define two constraint sets according to the following:

$$
\begin{aligned}
& \Phi_{i} \triangleq\left\{\mathbf{Q}_{i} \mid \operatorname{Tr}\left(\mathbf{Q}_{i}\right) \leqslant P_{T},\right. \\
& \left.\operatorname{Tr}\left(\mathbf{H}_{s, p, n} \mathbf{Q}_{i} \mathbf{H}_{s, p, n}^{H}\right) \leq P_{I, 1}, \mathbf{Q}_{i} \geq 0\right\}, \\
& \Psi_{i} \triangleq\left\{\mathbf{A}_{i} \mid \operatorname{Tr}\left[\mathbf{A}_{i}\left(\sigma_{r}^{2} \mathbf{I}_{N_{r}}+\mathbf{H}_{s r, i} \mathbf{Q}_{i} \mathbf{H}_{s r, i}^{H}\right) \mathbf{A}_{i}^{H}\right] \leq P_{R}, \mathbf{A}_{i} \geq 0,\right. \\
& \operatorname{Tr}\left\{\mathbf{H}_{i, p, n}\left[\mathbf{A}_{i}\left(\sigma_{r}^{2} \mathbf{I}_{N_{r}}+\mathbf{H}_{s r, i} \mathbf{Q}_{i} \mathbf{H}_{s r, i}^{H}\right) \mathbf{A}_{i}^{H}\right] \mathbf{H}_{i, p, n}^{H}\right\} \\
& \left.\leq P_{I, 2}\right\}
\end{aligned}
$$

for all $n \in \mathcal{S}_{\mathrm{PU}}$. It is easy to verify that (4) can be decomposed into three parts as follows:

$$
\max _{i \in \mathcal{S}_{R}}\left(\max _{\mathbf{Q}_{i} \in \Phi_{i}}\left(\max _{\mathbf{A}_{i} \in \Psi_{i}^{\prime}} R_{i}\left(\mathbf{Q}_{i}, \mathbf{A}_{i}\right)\right)\right) .
$$

Hence, solving (4) reduces to iteratively solve a subproblem with respect to $\mathbf{A}_{i}$, for all $i \in \mathcal{S}_{R}$ and $n \in \mathcal{S}_{\mathrm{PU}}$ (with $\mathbf{Q}_{i}$ fixed), then another subproblem with respect to $\mathbf{Q}_{i}$ (with $\mathbf{A}_{i}$ fixed, $\forall i \in \mathcal{S}_{R}$ and $n \in \mathcal{S}_{\mathrm{PU}}$ ), and finally a main problem with respect to $i$. Directly tackling problem (4) is intractable in general. However, we will exploit the inherent special structure to significantly reduce the problem complexity and convert it to an equivalent problem with scalar parameters. In what follows, we will first study the optimal structural properties of $\mathbf{A}_{i}$ and $\mathbf{Q}_{i}$. Then, we will reformulate (4).

\section{Optimal Power Allocation}

In the first subsection, the structure of the optimal amplification matrix in $i$ th $\mathrm{SU}$ for a given $\mathbf{Q}_{i}$ is investigated. Then, the optimal structure of $\mathbf{Q}_{i}$ is studied in the second subsection. Finally, based on these optimal structures, the problem in (4) is reformulated in third subsection.

3.1. The Structure of the Optimal Amplification Matrix. For now, we assume that $\mathbf{Q}_{i}$ is given. Let the eigenvalue decomposition of $\mathbf{H}_{s r, i} \mathbf{H}_{s r, i}^{H}$ and $\mathbf{H}_{r d, i}^{H} \mathbf{H}_{r d, i}$ be

$$
\begin{gathered}
\mathbf{H}_{s r, i} \mathbf{H}_{s r, i}^{H}=\mathbf{U}_{s r, i} \Sigma_{s r, i} \mathbf{U}_{s r, i}^{H}, \\
\mathbf{H}_{r d, i}^{H} \mathbf{H}_{r d, i}=\mathbf{V}_{r d, i} \Sigma_{r d, i} \mathbf{V}_{r d, i}^{H},
\end{gathered}
$$

where $\mathbf{U}_{s r, i}$ and $\mathbf{V}_{r d, i}$ are unitary matrices, $\Sigma_{s r, i}=\operatorname{diag}\left\{\alpha_{1}, \alpha_{2}\right.$, $\left.\ldots, \alpha_{N_{r}}\right\}$, with $\alpha_{l} \geq 0$, and $\Sigma_{r d, i}=\operatorname{diag}\left\{\beta_{1}, \beta_{2}, \ldots, \beta_{N_{r}}\right\}$ with $\beta_{l} \geq 0$. 
Proposition 2. The optimal amplification matrix of $S U i, \mathbf{A}_{i}$, has the following structure:

$$
\mathbf{A}_{i, o p t}=\mathbf{V}_{r d, i} \Lambda_{\mathbf{A}_{i}} \widetilde{\mathbf{U}}_{s r, i}^{H},
$$

where $\widetilde{\mathbf{U}}_{s r, i}$ is obtained by eigenvalue decomposition of $\widetilde{\mathbf{H}}_{s r, i}$ $\widetilde{\mathbf{H}}_{s r, i}^{H}$ and $\widetilde{\mathbf{H}}_{s r, i}=\mathbf{H}_{s r, i} \mathbf{Q}_{i}^{-1 / 2}$, that is, $\widetilde{\mathbf{H}}_{s r, i} \widetilde{\mathbf{H}}_{s r, i}^{H}$; that is, $\widetilde{\mathbf{H}}_{s r, i}$ $\widetilde{\mathbf{H}}_{s r, i}^{H}=\mathbf{H}_{s r, i} \widetilde{\mathbf{Q}}_{i} \mathbf{H}_{s r, i}^{H}=\widetilde{\mathbf{U}}_{s r, i} \widetilde{s}_{s r, i} \widetilde{\mathbf{U}}_{s r, i}^{H}$.

Proof. Please refer to the Appendix.

Let the singular value decomposition (SVD) of $\mathbf{H}_{s r, i}$ and $\mathbf{H}_{r d, i}$ be

$$
\begin{aligned}
& \mathbf{H}_{s r, i}=\mathbf{U}_{s r, i} \Lambda_{s r, i} \mathbf{V}_{s r, i}^{H}, \\
& \mathbf{H}_{r d, i}=\mathbf{U}_{r d, i} \Lambda_{r d, i} \mathbf{V}_{r d, i}^{H}
\end{aligned}
$$

which satisfies (7). Then exploiting (8), (9), and (3), the achievable data rates of the desired link can be written as

$$
\begin{aligned}
& R_{i}\left(\mathbf{Q}_{i}, \mathbf{A}_{i, \text { opt }}\right) \\
& \quad=\frac{1}{2} \log _{2}\left|\mathbf{I}_{N_{d}}+\Lambda_{r d, i}^{2} \Lambda_{\mathbf{A}_{i}}^{2} \widetilde{\Sigma}_{s r, i}\left(\sigma_{d}^{2} \mathbf{I}_{N_{d}}+\sigma_{r}^{2} \Lambda_{r d, i}^{2} \Lambda_{\mathbf{A}_{i}}^{2}\right)^{-1}\right| .
\end{aligned}
$$

According to (10), the achievable data rate in the desired SU link only depends on $\widetilde{\Sigma}_{s r, i}$ but not on $\widetilde{\mathbf{U}}_{s r, i}$. Then, it can be concluded that for any matrix $\widehat{\mathbf{Q}}_{i}$ which satisfies $\mathbf{H}_{s r, i} \widehat{\mathbf{Q}}_{i} \mathbf{H}_{s r, i}^{H}=$ $\widehat{\mathbf{U}}_{s r, i} \widetilde{\Sigma}_{s r, i} \widehat{\mathbf{U}}_{s r, i}^{H}$, the optimal data rate is the same as when the transmit covariance matrix in the desired link is any arbitrary matrix $\widetilde{\mathbf{Q}}_{i}$. Therefore, (8) can be written as

$$
\mathbf{A}_{i, \mathrm{opt}}=\mathbf{V}_{r d, i} \Lambda_{\mathbf{A}_{i}} \mathbf{U}_{s r, i}^{H} .
$$

3.2. The Structure of the Optimal Transmit Covariance Matrix. In this subsection, the optimal structure of the transmit covariance matrix of the desired link is determined.

Proposition 3. The structure of optimal transmit covariance matrix of SU TX is as follows:

$$
\mathbf{Q}_{i}=\mathbf{V}_{s r, i} \Lambda_{\mathbf{Q}_{i}} \mathbf{V}_{s r, i}^{H},
$$

where $\Lambda_{\mathbf{Q}_{i}}$ is a diagonal matrix and must be determined such that the achievable data rate in the desired link is maximized.

Proof. Suppose that $\widetilde{\Sigma}_{s r, i, 1}$ is $r \times r$, and then

$$
\begin{aligned}
\mathbf{H}_{s r, i} \widehat{\mathbf{Q}}_{i} \mathbf{H}_{s r, i}^{H} & =\widehat{\mathbf{U}}_{s r, i} \widetilde{\Sigma}_{s r, i} \widehat{\mathbf{U}}_{s r, i}^{H} \\
& =\left[\begin{array}{ll}
\widehat{\mathbf{U}}_{s r, i, 1} & \widehat{\mathbf{U}}_{s r, i, 2}
\end{array}\right]\left[\begin{array}{ll}
\widetilde{\Sigma}_{s r, i} & \\
& \mathbf{0}
\end{array}\right]\left[\begin{array}{ll}
\widehat{\mathbf{U}}_{s r, i, 1} & \widehat{\mathbf{U}}_{s r, i, 2}
\end{array}\right]^{H},
\end{aligned}
$$

where $\widehat{\mathbf{Q}}_{i}$ is any PSD (positive semidefinite.) matrix which satisfies $\mathbf{H}_{s r, i} \widehat{\mathbf{Q}}_{i} \mathbf{H}_{s r, i}^{H}=\widehat{\mathbf{U}}_{s r, i} \widetilde{s}_{s r, i} \widehat{\mathbf{U}}_{s r, i}^{H}$. Hence, the singular value decomposition of matrix $\mathbf{H}_{s r, i}$ with rank $r$ can be expressed as

$$
\begin{aligned}
\mathbf{H}_{s r, i} & =\mathbf{U}_{s r, i} \Lambda_{s r, i} \mathbf{V}_{s r, i}^{H} \\
& =\left[\begin{array}{ll}
\mathbf{U}_{s r, i, 1} & \mathbf{U}_{s r, i, 2}
\end{array}\right]\left[\begin{array}{ll}
\Lambda_{s r, i, 1} & 0 \\
& \mathbf{0}
\end{array}\right]\left[\begin{array}{ll}
\mathbf{V}_{s r, i, 1} & \mathbf{V}_{s r, i, 2}
\end{array}\right]^{H},
\end{aligned}
$$

where $\Lambda_{s r, i, 1}$ is $r \times r$. It can be shown that $\mathbf{U}_{s r, i, 1}$ is orthogonal to $\widehat{\mathbf{U}}_{s r, i, 2}$. Moreover, $\mathbf{U}_{s r, i, 2}$ is orthogonal to $\widehat{\mathbf{U}}_{s r, i, 1}$. The pseudoinverse of $\mathbf{H}_{s r, i}$ is denoted by $\mathbf{H}_{s r, i}^{+}$. Then, from $\mathbf{H}_{s r, i} \widehat{\mathbf{Q}}_{i} \mathbf{H}_{s r, i}^{H}=\widehat{\mathbf{U}}_{s r, i} \widetilde{\Sigma}_{s r, i} \widehat{\mathbf{U}}_{s r, i}^{H}$, we have

$$
\begin{aligned}
\mathbf{H}_{s r, i}^{+} \mathbf{H}_{s r, i} \widehat{\mathbf{Q}}_{i} \mathbf{H}_{s r, i}^{H} \mathbf{H}_{s r, i}^{+H} & \\
= & {\left[\begin{array}{ll}
\mathbf{V}_{s r, i, 1} & \mathbf{V}_{s r, i, 2}
\end{array}\right]\left[\begin{array}{ll}
\Lambda_{s r, i, 1}^{-1} & \mathbf{0}
\end{array}\right]\left[\begin{array}{ll}
\mathbf{U}_{s r, i, 1} & \mathbf{U}_{s r, i, 2}
\end{array}\right]^{H} } \\
& \times\left[\begin{array}{ll}
\widehat{\mathbf{U}}_{s r, i, 1} & \widehat{\mathbf{U}}_{s r, i, 2}
\end{array}\right]\left[\begin{array}{ll}
\widehat{\Sigma}_{s r, i, 1} & \mathbf{0}
\end{array}\right]\left[\begin{array}{ll}
\widehat{\mathbf{U}}_{s r, i, 1} & \widehat{\mathbf{U}}_{s r, i, 2}
\end{array}\right]^{H} \\
& \times\left[\begin{array}{ll}
\mathbf{U}_{s r, i, 1} & \mathbf{U}_{s r, i, 2}
\end{array}\right]\left[\begin{array}{ll}
\Lambda_{s r, i, 1}^{-1} & \mathbf{0}
\end{array}\right]\left[\begin{array}{ll}
\mathbf{V}_{s r, i, 1} & \mathbf{V}_{s r, i, 2}
\end{array}\right]^{H} \\
= & {\left[\begin{array}{ll}
\mathbf{V}_{s r, i, 1} & \mathbf{V}_{s r, i, 2}
\end{array}\right] } \\
& \times\left[\begin{array}{lll}
\Lambda_{s r, i, 1}^{-1} \mathbf{U}_{s r, i, 1}^{H} \widehat{\mathbf{U}}_{s r, i, 2} \widetilde{\Sigma}_{s r, i, 1} \widehat{\mathbf{U}}_{s r, i, 1} \mathbf{U}_{s r, i, 1} \Lambda_{s r, i, 1}^{-1} & \mathbf{0}
\end{array}\right] \\
& \times\left[\begin{array}{lll}
\mathbf{V}_{s r, i, 1} & \mathbf{V}_{s r, i, 2}
\end{array}\right]^{H} .
\end{aligned}
$$

It can be verified that $\mathbf{U}_{s r, i, 1}^{H} \widehat{\mathbf{U}}_{s r, i, 1}$ is a unitary matrix, because $\mathbf{U}_{s r, i}^{H} \widehat{\mathbf{U}}_{s r, i}$ is unitary. Recall that if $\mathbf{A}$ and $\mathbf{B}$ are two positive semidefinite $M \times M$ matrices with eigenvalues $\lambda_{i}(\mathbf{A})$ and $\lambda_{i}(\mathbf{B})$, arranged in the descending order, respectively, then

$$
\sum_{i=1}^{M} \lambda_{i}(\mathbf{A}) \lambda_{M+1-i}(\mathbf{B}) \leq \operatorname{Tr}(\mathbf{A B}) \leq \sum_{i=1}^{M} \lambda_{i}(\mathbf{A}) \lambda_{i}(\mathbf{B}) .
$$

Then, using the second inequality in (16) and knowing that $\mathbf{H}_{s r, i}^{H} \mathbf{H}_{s r, i}^{+H} \mathbf{H}_{s r, i}^{+} \mathbf{H}_{s r, i}$ is a project matrix with eigenvalues being only 1 and 0 , we have

$$
\begin{aligned}
\operatorname{Tr}\left(\mathbf{Q}_{i}\right) & \geq \operatorname{Tr}\left(\mathbf{H}_{s r, i}^{+} \mathbf{H}_{s r, i} \mathbf{Q}_{i} \mathbf{H}_{s r, i}^{H} \mathbf{H}_{s r, i}^{H+}\right) \\
& =\operatorname{Tr}\left(\Lambda_{s r, i, 1}^{-1} \mathbf{U}_{s r, i, 1}^{H} \widehat{\mathbf{U}}_{s r, i, 2} \widetilde{\Sigma}_{s r, i, 1} \widehat{\mathbf{U}}_{s r, i, 1} \mathbf{U}_{s r, i, 1} \Lambda_{s r, i, 1}^{-1}\right) .
\end{aligned}
$$

Also, using the first equality in (16), we can conclude that

$$
\operatorname{Tr}\left(\mathbf{Q}_{i}\right) \geq \operatorname{Tr}\left(\widetilde{\Sigma}_{s r, i, 1} \Lambda_{s r, i, 1}^{-2}\right) .
$$

Therefore, the structure of the optimal transmit covariance matrix in the desired link is given by

$$
\begin{aligned}
\mathbf{Q}_{\mathrm{opt}, i}= & {\left[\begin{array}{ll}
\mathbf{V}_{s r, i, 1} & \mathbf{V}_{s r, i, 2}
\end{array}\right]\left[\begin{array}{ll}
\widetilde{\Sigma}_{s r, i, 1} \Lambda_{s r, i, 1}^{-2} & \mathbf{0}
\end{array}\right] } \\
& \times\left[\begin{array}{ll}
\mathbf{V}_{s r, i, 1} & \mathbf{V}_{s r, i, 2}
\end{array}\right]^{H}
\end{aligned}
$$


which satisfies

$$
\mathbf{H}_{s r, i} \mathbf{Q}_{\mathrm{opt}, i} \mathbf{H}_{s r, i}^{H}=\mathbf{U}_{s r, i}\left[\begin{array}{ll}
\widetilde{\Sigma}_{s r, i, 1} & \\
& \mathbf{0}
\end{array}\right] \mathbf{U}_{s r, i}^{H}
$$

and the proposition is proved.

3.3. Problem Reformulation. In the previous subsection, we proved that the structure of the optimal amplification matrix in SU $i$ and transmit covariance matrix in the SU TX can be expressed as

$$
\begin{gathered}
\mathbf{A}_{i, \mathrm{opt}}=\mathbf{V}_{r d, i} \Lambda_{\mathbf{A}_{i}} \mathbf{U}_{s r, i}^{H}, \\
\mathbf{Q}_{i}=\mathbf{V}_{s r, i} \Lambda_{\mathbf{Q}_{i}} \mathbf{V}_{s r, i}^{H},
\end{gathered}
$$

where $\mathbf{H}_{s r, i}=\mathbf{U}_{s r, i} \Lambda_{s r, i} \mathbf{V}_{s r, i}^{H}$ and $\mathbf{H}_{r d, i}=\mathbf{U}_{r d, i} \Lambda_{r d, i} \mathbf{V}_{r d, i}^{H}$. Recall that the received signal in SU RX, due to the cooperation of $\mathrm{SU} i$, is given by

$$
\mathbf{y}_{d}=\mathbf{H}_{r d, i} \mathbf{A}_{i} \mathbf{H}_{s r, i} \mathbf{x}_{s, i}+\mathbf{H}_{r d, i} \mathbf{A}_{i} \mathbf{n}_{r, i}+\mathbf{n}_{d} .
$$

Using (21), $\mathbf{y}_{d}$ in (22) can be rewritten as

$$
\mathbf{y}_{d}=\mathbf{U}_{r d, i} \Lambda_{r d, i} \Lambda_{\mathbf{A}_{i}} \Lambda_{s r, i} \mathbf{V}_{s r, i}^{H} \mathbf{x}_{s, i}+\mathbf{U}_{r d, i} \Lambda_{r d, i} \Lambda_{\mathbf{A}_{i}} \mathbf{U}_{s r, i}^{H} \mathbf{n}_{r, i}+\mathbf{n}_{d} .
$$

Suppose that $\widetilde{\mathbf{y}}_{d}=\mathbf{U}_{r d, i}^{H} \mathbf{y}_{d}, \widetilde{\mathbf{x}}_{s, i}=\mathbf{V}_{s r, i}^{H} \mathbf{x}_{s, i}, \widetilde{\mathbf{n}}_{r, i}=\mathbf{U}_{s r, i}^{H} \mathbf{n}_{r, i}$, and $\widetilde{\mathbf{n}}_{d}=\mathbf{U}_{r d, i}^{H} \mathbf{n}_{d}$. Then,

$$
\widetilde{\mathbf{y}}_{d}=\Lambda_{r d, i} \Lambda_{\mathbf{A}_{i}} \Lambda_{s r, i} \widetilde{\mathbf{x}}_{s, i}+\Lambda_{r d, i} \Lambda_{\mathbf{A}_{i}} \widetilde{\mathbf{n}}_{r, i}+\widetilde{\mathbf{n}}_{d} .
$$

Clearly, the relay channel between the SU TX and SU RX has been decomposed into a set of parallel SISO subchannels. Therefore, the achievable data rates in the desired link, as a result of the cooperation of $\mathrm{SU} i$, can be expressed as

$$
R_{i}=\log _{2}\left|\mathbf{I}_{N_{d}}+\Lambda_{r d, i}^{2} \Lambda_{\mathbf{A}_{i}}^{2} \Lambda_{s r, i}^{2} \Lambda_{\mathbf{Q}_{i}}\left(\sigma_{r}^{2} \Lambda_{r d, i}^{2} \Lambda_{\mathbf{A}_{i}}^{2}+\sigma_{d}^{2} \mathbf{I}_{N_{d}}\right)^{-1}\right| .
$$

Suppose that the eigenvalue decomposition of $\mathbf{H}_{s, p, n}^{H} \mathbf{H}_{s, p, n}$ and $\mathbf{H}_{i, p, n}^{H} \mathbf{H}_{i, p, n}$ is

$$
\begin{aligned}
& \mathbf{H}_{s, p, n}^{H} \mathbf{H}_{s, p, n}=\mathbf{U}_{s, p, n} \Lambda_{s, p, n} \mathbf{U}_{s, p, n}^{H}, \\
& \mathbf{H}_{i, p, n}^{H} \mathbf{H}_{i, p, n}=\mathbf{U}_{i, p, n} \Lambda_{i, p, n} \mathbf{U}_{i, p, n}^{H}
\end{aligned}
$$

for all $n \in \mathcal{S}_{\mathrm{PU}}$. We further assume that

$$
\begin{gathered}
\Lambda_{\mathbf{A}_{i}}^{2}=\operatorname{diag}\left\{a_{1, i}, \ldots, a_{N_{r}, i}\right\}, \\
\Lambda_{s r, i}^{2}=\operatorname{diag}\left\{b_{1, i}, \ldots, b_{N_{r}, i}\right\}, \\
\Lambda_{r d, i}^{2}=\operatorname{diag}\left\{c_{1, i}, \ldots, c_{N_{r}, i}\right\}, \\
\Lambda_{s, p, n}=\operatorname{diag}\left\{d_{1, n}, \ldots, d_{N_{s}, n}\right\}, \quad \forall n \in \mathcal{S}_{\mathrm{PU}}, \\
\Lambda_{i, p, n}=\operatorname{diag}\left\{e_{1, i, n}, \ldots, e_{N_{r}, i, n}\right\}, \quad \forall n \in \mathcal{S}_{\mathrm{PU}}, \\
\Lambda_{\mathbf{Q}_{i}}=\operatorname{diag}\left\{q_{1, i}, \ldots, q_{N_{s}, i}\right\} .
\end{gathered}
$$

Then, using (27), (25) can be rewritten as

$$
R_{i}=\sum_{k=1}^{N_{r}} \log _{2}\left(1+\frac{a_{k, i} b_{k, i} c_{k, i} q_{k, i}}{\sigma_{r}^{2} a_{k, i} c_{k, i}+\sigma_{d}^{2}}\right) .
$$

Moreover, the transmit power constraint of the SU TX and $\mathrm{SU} i$ will become

$$
\begin{gathered}
\operatorname{Tr}\left(\mathbf{Q}_{i}\right) \leq P_{T} \Longrightarrow \sum_{k=1}^{N_{s}} q_{k, i} \leq P_{T}, \\
\operatorname{Tr}\left[\mathbf{A}_{i}\left(\sigma_{r}^{2} \mathbf{I}_{N_{r}}+\mathbf{H}_{s r, i} \mathbf{Q}_{i} \mathbf{H}_{s r, i}^{H}\right) \mathbf{A}_{i}^{H}\right] \\
=\operatorname{Tr}\left(\sigma_{r}^{2} \Lambda_{\mathbf{A}_{i}}^{2}+\Lambda_{s r i}^{2} \Lambda_{\mathbf{Q}_{i}} \Lambda_{\mathbf{A}_{i}}^{2}\right) \leq P_{R} \\
\Longrightarrow \sum_{k=1}^{N_{r}} a_{k, i}\left(\sigma_{r}^{2}+b_{k, i} q_{k, i}\right) \leq P_{R} .
\end{gathered}
$$

The interference constraint on PUs, due to transmission of SU TX, can be written as

$$
\begin{aligned}
\operatorname{Tr}\left(\mathbf{H}_{s, p, n} \mathbf{Q}_{i} \mathbf{H}_{s, p, n}^{H}\right) & =\operatorname{Tr}\left(\mathbf{H}_{s, p, n}^{H} \mathbf{H}_{s, p, n} \mathbf{Q}_{i}\right) \\
& =\operatorname{Tr}\left(\mathbf{U}_{s, p, n} \Lambda_{s, p, n} \mathbf{U}_{s, p, n}^{H} \mathbf{V}_{s r, i} \Lambda_{\mathbf{Q}_{i}} \mathbf{V}_{s r, i}^{H}\right) \\
& =\operatorname{Tr}\left(\mathbf{V}_{s r, i}^{H} \mathbf{U}_{s, p, n} \Lambda_{s, p, n} \mathbf{U}_{s, p, n}^{H} \mathbf{V}_{s r, i} \Lambda_{\mathbf{Q}_{i}}\right)
\end{aligned}
$$

for all $n \in \mathcal{S}_{\mathrm{PU}}$. Let $\mathbf{M}_{i, n}=\mathbf{V}_{s r, i}^{H} \mathbf{U}_{s, p, n}$. Then, (30) can be expressed as

$$
\operatorname{Tr}\left(\mathbf{M}_{i, n} \Lambda_{s, p, n} \mathbf{M}_{i}^{H} \Lambda_{\mathbf{Q}_{i}}\right)=\sum_{k=1}^{N_{s}}\left(\sum_{l=1}^{N_{s}}\left|m_{i, k, l, n}\right|^{2} d_{l}\right) q_{k, i},
$$

where $m_{i, k, l, n}$ denotes the element of $k$ th row and $l$ th column of matrix $\mathbf{M}_{i, n}$. Let $f_{k, i, n}=\sum_{l=1}^{N_{s}}\left|m_{i, k, l, n}\right|^{2} d_{l, n}$. Therefore, the interference constraint on PUs, due to transmitting by SU TX, is expressed by

$$
\operatorname{Tr}\left(\mathbf{H}_{s, p, n} \mathbf{Q}_{i} \mathbf{H}_{s, p, n}^{H}\right)=\sum_{k=1}^{N_{s}} f_{k, i, n} q_{k, i} \leq P_{I, 1} .
$$

The interference constraint on PUs, due to the cooperation of SU $i$ with SU TX, is written by

$$
\begin{aligned}
\operatorname{Tr}\left\{\mathbf{H}_{i, p, n} \mathbf{A}_{i}\left(\sigma_{r}^{2} \mathbf{I}_{N_{r}}+\mathbf{H}_{s r, i} \mathbf{Q}_{i} \mathbf{H}_{s r, i}^{H}\right) \mathbf{A}_{i}^{H} \mathbf{H}_{i, p, n}^{H}\right\} \\
=\operatorname{Tr}\left(\mathbf{V}_{r d, i}^{H} \mathbf{U}_{i, p, n} \Lambda_{i, p, n} \mathbf{U}_{i, p, n}^{H} \mathbf{V}_{r d, i} \Lambda_{\mathbf{A}_{i}}\right. \\
\left.\quad \times\left(\sigma_{r}^{2} \mathbf{I}_{N_{r}}+\Lambda_{s r, i} \Lambda_{\mathbf{Q}_{i}} \Lambda_{s r, i}\right) \mathbf{U}_{s r, i} \Lambda_{\mathbf{A}_{i}}\right) \leq P_{I, 2}
\end{aligned}
$$

for all $i \in \mathcal{S}_{R}$ and $n \in \mathcal{S}_{\mathrm{PU}}$. Let $\mathbf{S}_{i, n}=\mathbf{V}_{r d, i}^{H} \mathbf{U}_{i, p, n}$. The element of $k$ th row and $l$ th column of $\mathbf{S}_{i, n}$ is denoted by $s_{i, k, l, n}$. Hence, it can be shown that (33) can be rewritten as

$$
\sum_{k=1}^{N_{r}}\left(\sigma_{r}^{2}+b_{k, i} q_{k, i}\right) a_{k, i}\left(\sum_{l=1}^{N_{r}}\left|s_{i, k, l, n}\right|^{2} e_{l, i, n}\right) \leq P_{I, 2}
$$


Let $g_{k, i, n}=\sum_{l=1}^{N_{r}}\left|s_{i, k, l, n}\right|^{2} e_{l, i, n}$. Thus, the interference constraint on PUs, due to the cooperation of the selected SU in relaying the signals of the SU TX, is stated as

$$
\sum_{k=1}^{N_{r}}\left(\sigma_{r}^{2}+b_{k, i} q_{k, i}\right) a_{k, i} g_{k, i, n} \leq P_{I, 2}
$$

Note that, without loss of generality, it was assumed in (35) that $N_{s} \geq N_{r}$. If this assumption is not applicable, the only necessary modification in (35) is to change the upper limit of the inner sum to $\min \left(N_{r}, N_{s}\right)$, instead of $N_{r}$. Let $\mathbf{a}_{i}=$ $\left[a_{1, i}, \ldots, a_{N_{r}, i}\right]$ and $\mathbf{q}_{i}=\left[q_{1, i}, \ldots, q_{N_{s}, i}\right]$. Finally, the problem (4) can be expressed according to the following:

$$
\begin{aligned}
& \mathbf{q}_{i}^{*}, \mathbf{a}_{i}^{*}=\underset{\mathbf{q}_{i}, \mathbf{a}_{i}}{\arg \max } \sum_{k=1}^{N_{r}} \log _{2}\left(1+\frac{a_{k, i} b_{k, i} c_{k, i} q_{k, i}}{\sigma_{r}^{2} a_{k, i} c_{k, i}+\sigma_{d}^{2}}\right), \\
& i=\underset{i}{\arg \max } \sum_{k=1}^{N_{r}} \log _{2}\left(1+\frac{a_{k, i}^{*} b_{k, i} c_{k, i} q_{k, i}^{*}}{\sigma_{r}^{2} a_{k, i}^{*} c_{k, i}+\sigma_{d}^{2}}\right), \\
& \text { subject to } \sum_{k=1}^{N_{s}} q_{k, i} \leq P_{T} \text {, } \\
& \sum_{k=1}^{N_{r}} a_{k, i}\left(\sigma_{r}^{2}+b_{k, i} q_{k, i}\right) \leq P_{R} \\
& \sum_{k=1}^{N_{s}} f_{k, i, n} q_{k, i} \leq P_{I, 1}, \quad \forall n \in \mathcal{S}_{\mathrm{PU}}, \\
& \sum_{k=1}^{N_{r}} g_{k, i, n} a_{k, i}\left(\sigma_{r}^{2}+b_{k, i} q_{k, i}\right) \leq P_{I, 2}, \quad \forall n \in \mathcal{S}_{\mathrm{PU}} .
\end{aligned}
$$

Let $h_{k, i}=a_{k, i}\left(\sigma_{r}^{2}+b_{k, i} q_{k, i}\right)$. By some simple derivations, the problem in (36) is equivalent to

$$
\begin{aligned}
& \mathbf{q}_{i}^{*}, \mathbf{h}_{i}^{*}=\underset{\mathbf{q}_{i} \mathbf{h}_{i}}{\arg \max } \sum_{k=1}^{N_{r}} \log _{2} \frac{\left(1+c_{k, i} h_{k, i} / \sigma_{d}^{2}\right)\left(1+b_{k, i} q_{k, i} / \sigma_{r}^{2}\right)}{1+c_{k, i} h_{k, i} / \sigma_{d}^{2}+b_{k, i} q_{k, i} / \sigma_{r}^{2}}, \\
& i=\underset{i}{\arg \max } \sum_{k=1}^{N_{r}} \log _{2} \frac{\left(1+c_{k, i} h_{k, i}^{*} / \sigma_{d}^{2}\right)\left(1+b_{k, i} q_{k, i}^{*} / \sigma_{r}^{2}\right)}{1+c_{k, i} h_{k, i}^{*} / \sigma_{d}^{2}+b_{k, i} q_{k, i}^{*} / \sigma_{r}^{2}}, \\
& \text { subject to } \sum_{k=1}^{N_{s}} q_{k, i} \leq P_{T}, \\
& \sum_{k=1}^{N_{r}} h_{k, i} \leq P_{R}, \\
& \sum_{k=1}^{N_{s}} f_{k, i, n} q_{k, i} \leq P_{I, 1}, \quad \forall n \in \mathcal{S}_{\mathrm{PU}}, \\
& \sum_{k=1}^{N_{r}} g_{k, i, n} h_{k, i} \leq P_{I, 2}, \quad \forall n \in \mathcal{S}_{\mathrm{PU}} .
\end{aligned}
$$

\section{Optimization Algorithm}

In this section, we develop approaches for joint relay selection and power allocation in cooperative cognitive radio networks. At first, we provide an optimal approach and then develop a low complexity suboptimal approach.

4.1. Optimal Approach. Using the Lagrange multipliers method [27], the Lagrange function for (37) is given by

$$
\begin{aligned}
\mathscr{L}\left(\mathbf{h}_{i}, \mathbf{q}_{i}, \lambda_{1}, \lambda_{2}, \lambda_{3, n}, \lambda_{4, n}\right) \\
=-\sum_{k=1}^{N_{r}} \log _{2} \frac{\left(1+c_{k, i} h_{k, i} / \sigma_{d}^{2}\right)\left(1+b_{k, i} q_{k, i} / \sigma_{r}^{2}\right)}{1+c_{k, i} h_{k, i} / \sigma_{d}^{2}+b_{k, i} q_{k, i} / \sigma_{r}^{2}} \\
+\lambda_{1}\left(\sum_{k=1}^{N_{s}} q_{k, i}-P_{T}\right)+\lambda_{2}\left(\sum_{k=1}^{N_{r}} h_{k, i}-P_{R}\right) \\
+\sum_{n=1}^{N_{\mathrm{PU}}} \lambda_{3, n}\left(\sum_{k=1}^{N_{r}} f_{k, i, n} q_{k, i}-P_{I, 1}\right) \\
+\sum_{n=1}^{N_{\mathrm{PU}}} \lambda_{4, n}\left(\sum_{k=1}^{N_{r}} g_{k, i, n} h_{k, i}-P_{I, 2}\right),
\end{aligned}
$$

where $\lambda_{1}, \lambda_{2}, \lambda_{3, n}$, and $\lambda_{4, n}$ are the Lagrange multipliers and $\forall n \in \mathcal{S}_{\mathrm{PU}}$. According to the KKT conditions, we have

$$
\begin{gathered}
\lambda_{1} \geq 0, \quad \lambda_{2} \geq 0, \quad \lambda_{3, n} \geq 0, \quad \lambda_{4, n} \geq 0, \\
q_{l, i} \geq 0, \quad h_{k, i} \geq 0, \quad l=1, \ldots, N_{s}, \quad k=1, \ldots, N_{r} \\
\lambda_{1}\left(\sum_{k=1}^{N_{s}} q_{k, i}-P_{T}\right)=0, \\
\lambda_{2}\left(\sum_{k=1}^{N_{s}} h_{k, i}-P_{R}\right)=0, \\
\lambda_{3, n}\left(\sum_{k=1}^{N_{s}} f_{k, i n} q_{k, i}-P_{I, 1}\right)=0, \\
\lambda_{4, n}\left(\sum_{k=1}^{N_{r}} g_{k, i, n} h_{k, i}-P_{I, 2}\right)=0, \\
\frac{\partial \mathscr{L}}{\partial q_{k, i}}=0, \quad k=1, \ldots, N_{s}, \\
\frac{\partial \mathscr{L}}{\partial h_{k, i}}=0, \quad k=1, \ldots, N_{r}
\end{gathered}
$$


for all $n \in \mathcal{S}_{\mathrm{PU}}$ and $i \in \mathcal{S}_{R}$. It can be shown that $h_{k, i}$ and $q_{k, i}$ can be obtained using the following equations:

$$
\begin{aligned}
q_{k, i}=\frac{\sigma_{r}^{2}}{2 b_{k, i}}[ & \sqrt{\frac{c_{k, i}^{2}}{\sigma_{d}^{4}} h_{k, i}^{2}-\frac{4 b_{k, i} c_{k, i}}{\left(\lambda_{1}+f_{k, i, n} \lambda_{3}\right) \sigma_{r}^{2} \sigma_{d}^{2} \ln 2} h_{k, i}} \\
& \left.-\left(2+\frac{c_{k, i}}{\sigma_{d}^{2}} h_{k, i}\right)\right]^{+}, \\
h_{k, i}=\frac{\sigma_{d}^{2}}{2 c_{k, i}}[ & \sqrt{\frac{b_{k, i}^{2}}{\sigma_{r}^{4}} q_{k}^{2}-\frac{4 b_{k, i} c_{k, i}}{\left(\lambda_{2}+g_{k, i, n} \lambda_{4}\right) \sigma_{r}^{2} \sigma_{d}^{2} \ln 2} q_{k}} \\
& \left.-\left(2+\frac{b_{k, i}}{\sigma_{r}^{2}} q_{k, i}\right)\right]^{+},
\end{aligned}
$$

where $[\cdot]^{+}=\max (\cdot, 0)$. Using dual-domain and subgradient methods [28], we can further obtain $\lambda_{1}, \lambda_{2}, \lambda_{3, n}$, and $\lambda_{4, n}$ through iteration,

$$
\begin{gathered}
\lambda_{1}^{(m+1)}=\left[\lambda_{1}^{(m)}+\mu^{(m)}\left(\sum_{k=1}^{N_{s}} q_{k, i}^{(m)}-P_{T}\right)\right]^{+}, \\
\lambda_{2}^{(m+1)}=\left[\lambda_{2}^{(m)}+\mu^{(m)}\left(\sum_{k=1}^{N_{r}} h_{k, i}^{(m)}-P_{R}\right)\right]^{+}, \\
\lambda_{3, n}^{(m+1)}=\left[\lambda_{3, n}^{(m)}+\mu^{(m)}\left(\sum_{k=1}^{N_{r}} f_{k, i, n} q_{k, i}^{(m)}-P_{I, 1}\right)\right]^{+}, \\
\lambda_{4, n}^{(m+1)}=\left[\lambda_{4, n}^{(m)}+\mu^{(m)}\left(\sum_{k=1}^{N_{r}} g_{k, i, n} h_{k, i}^{(m)}-P_{I, 2}\right)\right]^{+}
\end{gathered}
$$

for all $n \in \mathcal{S}_{\mathrm{PU}}$, where $m$ is the iteration index and $\mu^{(m)}$ is a sequence of scalar step sizes. Once $\lambda_{1}, \lambda_{2}, \lambda_{3, n}$, and $\lambda_{4, n}$ are obtained, we can get the optimal power allocation matrices $\mathbf{Q}_{i}$ and $\mathbf{A}_{i}$ and the corresponding achievable data rate $R_{i}$ when the $i$ th SU acts as the relay for the SU TX. Repeating the above procedures at all SUs, we then find the one with the maximum achievable data rate.

4.2. Low Complexity Approach. The optimal approach performs joint opportunistic relay selection and power allocation and results in the maximum data rate. However, the optimal approach is with very high complexity. Here, we aim to develop an alternate low complexity suboptimal approach for problem (37). At first, we assume that the available source power is distributed uniformly over the spatial modes; that is, $q_{i}^{\text {uni }}=P_{T} / N_{s}$. Similar assumption applies for $h_{k, i}$ 's $(k=$ $\left.1, \ldots, N_{r}\right)$; that is, $h_{i}^{\text {uni }}=P_{R} / N_{r}$. Also assume that the interference introduced to the PU by each spatial mode of SU TX is equal and hence the maximum allowable power that can be allocated to the $k$ th mode is $q_{k, i}^{\max }=P_{I, 1} / N_{s} f_{k, i}^{\max }$, where $f_{k, i}^{\max }=\max _{n \in \mathcal{S}_{\mathrm{PU}}} f_{k, i, n}$. Therefore, the allocated power to the $k$ th mode in the SU TX, intended for SU $i$, is $q_{k, i}^{*}=$ $\min \left\{q_{i}^{\text {uni }}, q_{k, i}^{\max }\right\}$ for $k=1, \ldots, N_{s}$ and $\forall i \in \mathcal{S}_{R}$. Similarly, we assume that the interference introduced to the PU by each spatial mode of SU $i$ is equal. Therefore, it can be concluded that $h_{k, i}^{\max }=P_{I, 2} / N_{r} g_{k, i}^{\max }$, where $g_{k, i}^{\max }=\max _{n \in \mathcal{S}_{\mathrm{PU}}} g_{k, i, n}$. Therefore, the power allocation in the SU $i$ is given by $h_{k, i}^{*}=$ $\min \left\{h_{i}^{\text {uni }}, h_{k, i}^{\max }\right\}$ for $k=1, \ldots, N_{r}$ and $\forall i \in \mathcal{S}_{R}$. Afterwards, the SU $i$ is selected as the cooperative relay such that the following is maximized:

$$
i=\arg \max _{i} \sum_{k=1}^{N_{r}} \log _{2} \frac{\left(1+c_{k, i} h_{k, i}^{*} / \sigma_{d}^{2}\right)\left(1+b_{k, i} q_{k, i}^{*} / \sigma_{r}^{2}\right)}{1+c_{k, i} h_{k, i}^{*} / \sigma_{d}^{2}+b_{k, i} q_{k, i}^{*} / \sigma_{r}^{2}} .
$$

After determining the cooperative SU, we calculate the optimal transmit covariance matrix, $\mathbf{Q}_{i}$, and amplification matrix, $\mathbf{A}_{i}$, using the approach provided in the optimal approach subsection. As we can see from the simulation results, this approach is almost as good as the optimal approach. However, it is with much lower complexity.

A comparison between the computational complexity of the optimal and suboptimal methods is presented here. For the optimal solution derived in the previous section, $2\left(N_{\mathrm{PU}}+\right.$ 1) dual variables are updated in every iteration. Using these values, $N_{r}^{2}$ function evaluations are performed to find the power allocation. Therefore, the optimal solution derived in the previous section has a complexity of $\mathcal{O}\left(N_{r}^{2}+2\left(N_{\mathrm{PU}}+1\right) T\right)$, where $T$ is the number of iterations required to converge, which is usually high [28]. In the suboptimal scheme, every spatial mode in the source side requires not more than $\left(N_{\mathrm{SU}}+\right.$ $N_{\mathrm{SU}} N_{r}$ ) function evaluations. Therefore, the complexity of the proposed algorithm is $\mathcal{O}\left(N_{\mathrm{SU}} N_{r}\right)$.

\section{Outage Analysis}

In order to analyze the outage behaviour of the proposed system, we consider the scenario where the PU transmitters, $\mathrm{PU} \mathrm{TX}_{1}, \ldots, \mathrm{PU} \mathrm{TX}_{N_{\mathrm{pU}}}$, randomly communicate with their respective receivers, $\mathrm{PU} \mathrm{RX}_{1}, \ldots, \mathrm{PU} \mathrm{RX}_{N_{\mathrm{PU}}}$. The interval between two transmissions of PUs and the duration of one PU transmission are assumed being random and obeying exponential distribution with two parameters $\theta$ and $\tau$, respectively. According to queuing theory, the probability of the absence of the PUs, $P(A)$, and the probability of the presence of the PUs, $P(\bar{A})$, can be expressed, respectively, as $P(A)=$ $\left(\sum_{n=0}^{N_{\mathrm{PU}}}\left(N_{\mathrm{PU}} ! /\left(N_{\mathrm{PU}}-n\right) !\right)(\theta / \tau)^{n}\right)^{-1}=\alpha$ and $P(\bar{A})=1-\alpha$.

In order to facilitate the analysis of outage, we modify the system model as explained below. First of all, we assume that the transmit signal at the SU TX is white and thereby $\mathbf{Q}=\rho \mathbf{I}_{N_{s}}$, where $\mathbf{I}_{N_{s}}$ represents the $N_{s} \times N_{s}$ identity matrix and $\rho N_{s}$ is the transmit power of the SU TX. Moreover, the cooperation strategy of the selected SU is assumed to be decode-and-forward (DF) strategy. This strategy switch is intended for some reasons, which among them is to obtain a lower bound for the outage capacity of the desired MIMO link. Meanwhile, this assumption facilitates the analysis of the outage probability analysis, as will be shown below. 
In the first time-slot, the spectrum sensing is used to detect whether the PUs are absent. When the PUs are absent, SU TX transmits data to SU RX directly. When the PUs are present, the transmit power of SU TX, $\rho N_{s}$, should be limited. However, if $\rho N_{s}$ is too low, the data from SU TX cannot reach SU RX. Thus, we use cooperative relaying to transmit signal from SU TX to SU RX through the best relay which is selected out of available SUs, as described in the previous section. In the sequel, we derive the approximate outage probabilities of the desired SU link, when the PUs are present and when no PUs transmit signals or, in other words, the PUs are absent.

5.1. Absence of PUs. We firstly assume that no PU link is transmitting signal. Hence, the SU TX communicates directly with the SU RX and the received signal in the SU RX can be written as

$$
\mathbf{y}_{d}=\mathbf{H}_{s d} \mathbf{x}_{s}+\mathbf{n}_{d}
$$

Based on the assumptions expressed at the beginning of this section, the achievable data rates of the desired link using the direct channel are given by

$$
R^{D}=\log _{2}\left|\mathbf{I}_{N_{d}}+\frac{\rho}{\sigma_{d}^{2}} \mathbf{H}_{s d} \mathbf{H}_{s d}^{H}\right|
$$

where $\mathbf{H}_{s d} \in \mathbb{C}^{N_{d} \times N_{s}}$ represents the direct channel in the desired link. It is obvious that the achievable data rates in the desired link, $R^{D}$, are a random variable which depends on the random nature of $\mathbf{H}_{s d}$. In a full-rank system, (44) can be simplified by using the singular value decomposition (SVD) as

$$
R^{D}=\sum_{m=1}^{N_{d}} \log _{2}\left(1+\frac{\rho}{\sigma_{d}^{2}} \lambda_{s d, m}\right)
$$

where $\lambda_{s d, m}, m=1, \ldots, N_{d}$ are the nonnegative eigenvalues of the channel covariance matrix $\mathbf{H}_{s d} \mathbf{H}_{s d}^{H}$. The joint pdf of $\lambda_{s d, m}, m=1, \ldots, N_{d}$ is given by [29]

$$
\begin{aligned}
p\left(\lambda_{s d, 1}, \ldots, \lambda_{s d, N_{d}}\right)= & \left(N_{d} ! K_{N_{d}, N_{s}}\right)^{-1} \exp \left(-\sum_{m=1}^{N_{d}} \lambda_{s d, m}\right) \\
& \times\left(\prod_{m=1}^{N_{d}} \lambda_{s d, m}^{N_{r}-N_{d}}\right) \\
& \times\left(\prod_{m<n}\left(\lambda_{s d, m}-\lambda_{s d, n}\right)^{2}\right),
\end{aligned}
$$

where $K_{N_{d}, N_{s}}$ is a normalizing factor. To ensure QoS for the desired link, it needs to support a minimum rate. When the instantaneous achievable data rate is less than the minimum rate, $R_{\min }$, an outage event occurs. In quasistatic fading, since the fading coefficients are constant over the whole frame, we cannot average them with an ergodic measure. In such an event, Shannon capacity does not exist in the ergodic sense [30]. The probability of such an event is normally referred to as outage probability. As described in [31], the distribution of the random achievable data rate can be viewed as Gaussian when the number of transmit and/or receive antennas goes to infinity. It is also a very good approximation for even small $N_{d}$ and $N_{s}$; for example, $N_{s}=N_{d}=2$ [31]. As such, for a sufficiently large $N_{d}$ and $N_{s}$, the achievable data rate of the desired link is approximated as [31]

$$
R^{D} \longrightarrow \mathcal{N}\left(N_{d} \log _{2}(1+\rho), \frac{N_{d} \rho^{2}}{(\ln 2)^{2} N_{s}(1+\rho)^{2}}\right)
$$

Then, we proceed by considering the distribution of the achievable data rate in the desired link as Gaussian with the pdf given in (47). Consequently, it can be shown that the outage probability of the desired link in the absence of the PUs can be written as

$$
\begin{aligned}
P_{\text {out }}^{D} & =P\left(R^{D}<R_{\min }\right) \\
& =Q\left(\frac{N_{d} \log _{2}(1+\rho)-R_{\min }}{\sqrt{N_{d} \rho^{2} /(\ln 2)^{2} N_{r}(1+\rho)^{2}}}\right),
\end{aligned}
$$

where $Q(\cdot)$ denotes the $Q$-function. We emphasize that (48) provides only an approximation of the outage probability in the desired link in the absence of the PUs.

5.2. Presence of PUs. As described in the previous section, when PUs transmit signals, the direct communication in the desired link must be avoided and the cooperation of the best SU is employed instead. The received signal in the SU RX using the cooperation of $i$ th SU can be expressed as

$$
\mathbf{y}_{d}=\mathbf{H}_{r d, i} \mathbf{x}_{s, i}+\mathbf{n}_{d}
$$
by

Thus, the achievable data rates of the desired link is given

$$
R_{i}^{C}=\frac{1}{2} \log _{2}\left|\mathbf{I}_{N_{d}}+\frac{\rho}{\sigma_{d}^{2}} \mathbf{H}_{r d, i} \mathbf{H}_{r d, i}^{H}\right| .
$$

The coefficient $1 / 2$ is due to the cooperative transmission and the transmission in two consecutive time-slots. Similar to the previous subsection, it can be concluded that for the case of present PUs, the achievable data rates in the desired link, $R_{i}^{C}$, can be expressed as

$$
R_{i}^{C}=\frac{1}{2} \sum_{m=1}^{N_{d}} \log _{2}\left(1+\frac{\rho}{\sigma_{d}^{2}} \lambda_{r d, i, m}\right),
$$


where $\lambda_{r d, i, m}, m=1, \ldots, N_{d}$ are the nonnegative eigenvalues of the channel covariance matrix $\mathbf{H}_{r d, i} \mathbf{H}_{r d, i}^{H}$. The joint pdf of $\lambda_{r d, i, m}, m=1, \ldots, N_{d}$ is given by [29]

$$
\begin{aligned}
p\left(\lambda_{r d, i, 1}, \ldots, \lambda_{r d, i, N_{d}}\right)= & \left(\mathrm{N}_{d} ! K_{N_{d}, N_{r}}\right)^{-1} \exp \left(-\sum_{m=1}^{N_{d}} \lambda_{r d, i, m}\right) \\
& \times\left(\prod_{m=1}^{N_{d}} \lambda_{r d, i, m}^{N_{r}-N_{d}}\right) \\
& \times\left(\prod_{m<n}\left(\lambda_{r d, i, m}-\lambda_{r d, i, n}\right)^{2}\right)
\end{aligned}
$$

where $K_{N_{d}, N_{r}}$ is a normalizing factor. Once again and similar to the previous discussions, the achievable data rate of the desired link is approximated as [31]

$$
R_{i}^{C} \longrightarrow \mathcal{N}\left(N_{d} \log _{2}(1+\rho), \frac{N_{d} \rho^{2}}{4(\ln 2)^{2} N_{r}(1+\rho)^{2}}\right) .
$$

Note that the coefficient $1 / 4$ in the variance of the pdf in (53) is due to the multiplication of $1 / 2$ in (51). Therefore, the outage probability of the desired link in the presence of the PUs can be written as

$$
\begin{aligned}
P_{\text {out }}^{C, i} & =P\left(R_{i}^{C}<R_{\min }\right) \\
& =Q\left(\frac{N_{d} \log _{2}(1+\rho)-R_{\min }}{\sqrt{N_{d} \rho^{2} / 4(\ln 2)^{2} N_{r}(1+\rho)^{2}}}\right) .
\end{aligned}
$$

5.3. The Outage Probability. In this subsection, the outage probability of the system is obtained. However, in the case that the DF cooperation strategy is employed and the PUs are present, another possible case in the system is when no SU can decode the signal from SU TX. This may be due to detrimental effects of fading and path loss in the link from the SU TX to SUs. In this case, the SU TX indispensably transmits data to SU TX directly with limited power $\rho^{\prime} N_{s}$ in order not to disturb the PUs. Assume that $\Delta_{u}$ is a nonempty subset of the $N_{\mathrm{SU}}$ secondary users who can decode the data of SU TX; that is, $\Delta_{u} \subseteq \mathcal{S}_{R}$, and assume that $\bar{\Delta}_{u}$ is the complementary set of $\Delta_{u}$. Suppose that $\phi$ is a null set. Then, the probability of no existing SU to decode the data of SU TX, $P_{\text {out }}^{\phi}$, can be written as

$$
P_{\mathrm{out}}^{\phi}=P(\Delta=\phi)=\prod_{m=1}^{N_{\mathrm{SU}}} P_{\mathrm{out}}^{R, m}
$$

and $P_{\text {out }}^{R, m}$, where $m \in \mathcal{S}_{R}$ denotes the outage probability in the link from SU TX to the SUs in the first time-slot. Similar to previous subsections, a good approximate for $P_{\text {out }}^{R, m}$ can be obtained as

$$
P_{\text {out }}^{R, m}=Q\left(\frac{N_{r} \log _{2}\left(1+\rho^{\prime}\right)-R_{\min }}{\sqrt{N_{r} \rho^{\prime 2} /(\ln 2)^{2} N_{s}\left(1+\rho^{\prime}\right)^{2}}}\right) .
$$

In the following theorem, we derive the outage probability of the desired SU link.

Theorem 4. The outage probability of the desired SU link is

$$
P_{\text {out }}=(1-\alpha)\left(P_{\text {out }}^{\phi}+\sum_{u=1}^{2^{N_{S U}-1}} P\left(\Delta=\Delta_{u}\right) P_{\text {out }}^{\Delta_{u}}\right)+\alpha P_{\text {out }}^{D} \text {, }
$$

where $P_{\text {out }}^{\Delta_{u}}$ is the outage probability of the desired link in the presence of PUs and when the one SUs in the subset $\Delta_{u}$ is cooperating with desired link.

Proof. Consider the case that the PUs are present. Then, the probability of event $\left\{\Delta=\Delta_{u}\right\}$, that is, there exist some SUs which can decode the signal from SU TX, can be written as

$$
P\left(\Delta=\Delta_{u}\right)=\left(\prod_{m \in \Delta_{u}}\left(1-P_{\text {out }}^{R, m}\right)\right)\left(\prod_{m \in \bar{\Delta}_{u}} P_{\text {out }}^{\mathrm{R}, m}\right)=\beta_{u} .
$$

The outage probability of the desired link in the presence of PUs and when the one SUs in the subset $\Delta_{u}$ is cooperating with desired link is given by

$$
P_{\text {out }}^{\Delta_{u}}=\prod_{i \in \Delta_{u}} P_{\text {out }}^{C, i}
$$

Then, the outage probability of the desired SU link in the presence of the PU signals can be written as

$$
P_{\text {out }}^{\bar{A}}=P_{\text {out }}^{\phi}+\sum_{u=1}^{2^{N_{\mathrm{SU}}-1}} P\left(\Delta=\Delta_{u}\right) P_{\text {out }}^{\Delta_{u}}
$$

Finally, it can be concluded that the outage probability of the desired link is given by

$$
\begin{aligned}
P_{\text {out }} & =P(\bar{A}) P_{\text {out }}^{\bar{A}}+P(A) P_{\text {out }}^{A} \\
& =(1-\alpha)\left(P_{\text {out }}^{\phi}+\sum_{u=1}^{2_{\text {SU }}-1} P\left(\Delta=\Delta_{u}\right) P_{\text {out }}^{\Delta_{u}}\right)+\alpha P_{\text {out }}^{D},
\end{aligned}
$$

where $P_{\text {out }}^{D}$ is the outage probability of the desired link, when the PUs are absent, and is given in (48) and the proof is complete in this way.

\section{Simulation Results}

In this section, the performance of the proposed MIMO cooperative cognitive radio system is evaluated using simulations. More specifically, we evaluate the performance of the proposed low complexity approach and compare it with the optimal approach. For better comprehending the merit of the proposed low complexity approach (LCA), we will also compare the proposed approach with the approaches using random cooperative SU selection with optimal power 


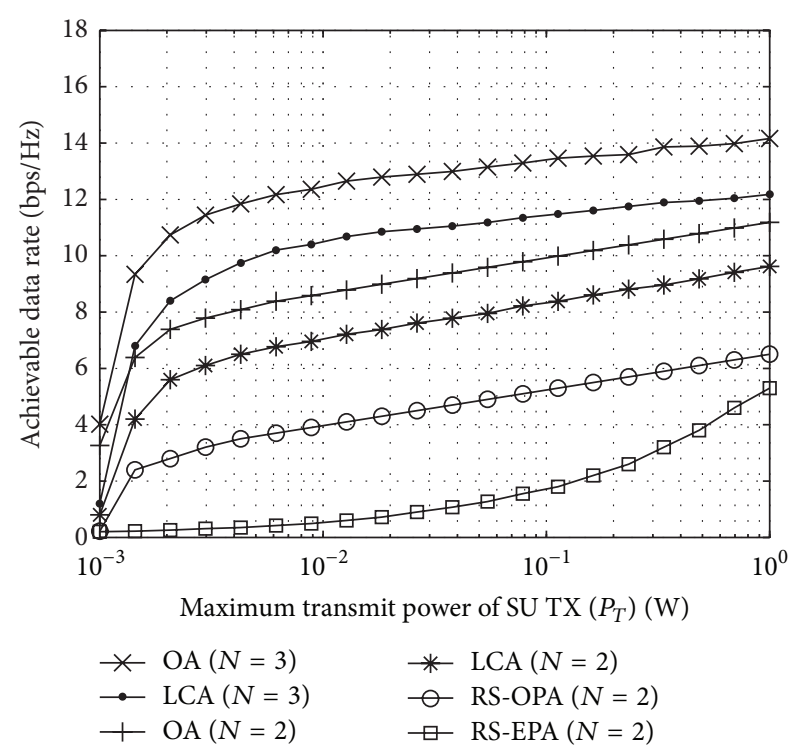

FIgURE 2: Achievable data rate in the desired link versus the maximum transmit power of SU TX $\left(P_{T}\right)$.

allocation matrices (transmit covariance matrix and amplification matrix), referred to as RS-OPA (random SU-optimal power allocation) and nonoptimal power allocation; that is, the amplification matrix of the randomly selected SU and the transmit covariance matrix are obtained as described in Section 4.2, respectively, which is referred to as RSEPA (random SU-equal power allocation). The simulation assumptions are as follows, otherwise stated.

(i) All users are assumed to be equipped with the same number of antennas, denoted by $N$.

(ii) We set interference limits, $P_{I, 1}=P_{I, 2}=0.01 \mathrm{~W}$, otherwise stated.

(iii) There exist 5 PU pairs in the system, otherwise stated.

(iv) The elements of the channel matrices follow a Rayleigh distribution and are independent of each other.

(v) The SUs are uniformly located between the SU TX and SU RX.

(vi) The path-loss exponent is 4 , and the standard deviation of shadowing is $6 \mathrm{~dB}$.

(vii) The number of existing SUs in the system, $N_{\mathrm{SU}}$, is 20, otherwise stated.

(viii) The level of noise is assumed identical in the system and $\sigma_{r}^{2}=\sigma_{d}^{2}=10^{-6} \mathrm{~W} / \mathrm{Hz}$.

The achievable data rate in the desired SU link versus the maximum transmit power of SU TX for different number of antennas and various scenarios is shown in Figure 2. The maximum transmit power of each $\mathrm{SU} i$, for all $i \in$ $\mathcal{S}_{R}$, is $P_{R}=0.2 \mathrm{~W}$. Using the low complexity approach (LCA), $50 \%$ achievable data rate gain over the RS-OPA is obtained, when $N=2$. Moreover, LCA leads to only $14 \%$

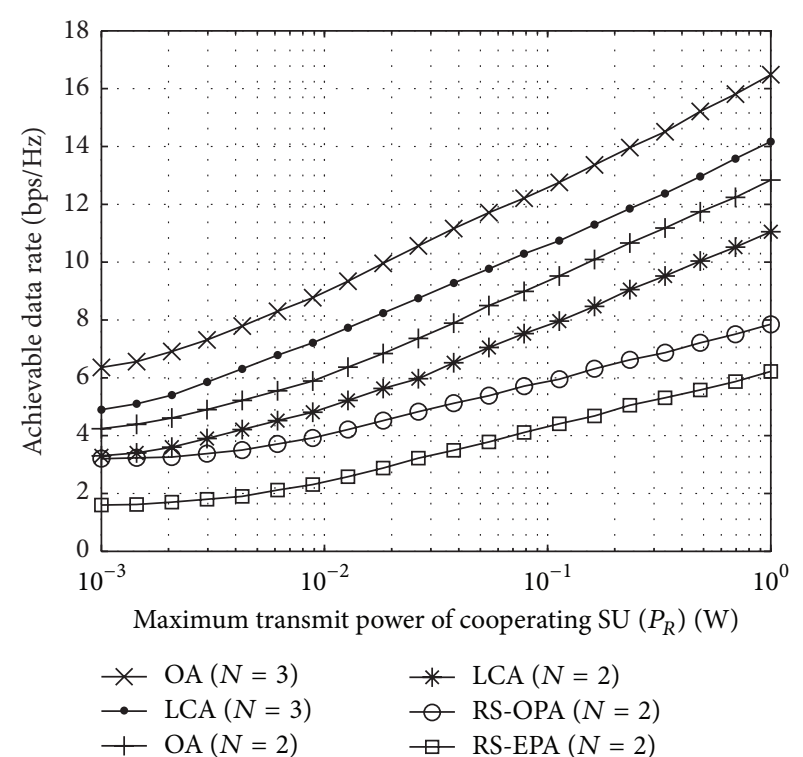

FIgURE 3: Achievable data rate in the desired link versus the maximum transmit power of cooperating SU $\left(P_{R}\right)$.

data rate degradation compared with $\mathrm{OA}$, with much lower complexity. When $P_{T}$ is small, the achievable data rate in the desired SU link increases rapidly with $P_{T}$. However, for large amounts of $P_{T}$, due to restrictions by the interference limits, the achievable data rate is not sensitive to the $P_{T}$. As another observation, it can also be seen that the random SU and optimal power allocation scheme (RS-OPA) achieves a significant gain in the achievable data rate over the random SU and nonoptimal (equal) power allocation scheme (RSEPA), especially when $P_{T}$ is small.

The achievable data rate of the desired SU link versus the maximum transmit power of the cooperating $\mathrm{SU}\left(P_{R}\right)$ is depicted in Figure 3. The maximum transmit power of the SU TX is fixed at $P_{T}=0.4 \mathrm{~W}$ and the number of existing SUs in the secondary network, $N_{\mathrm{SU}}$, is 20 . It can be concluded that the achievable data rate in the system is mainly determined by the transmit power of the cooperating SU, when the maximum transmit power of the SU TX is a constant.

As shown in Figure 4, the achievable data rate in the desired link grows with the number of the existing SUs in the CR network. However, this growth saturates from a particular number of SUs which shows that the increasing number of existing SUs will not necessarily result in the similar increase in the data rate of the desired link. Moreover, deploying larger number of antennas in users, that is, larger $N$, compensates for the less maximum transmit power of SU TX and the cooperating relay. It must also be noted that the achievable data rate in the system is increased with the number of existing SUs due to multiuser diversity.

As a final note, all the simulation results are indicating the undeniable effect of the deploying multiple antennas at the SUs and cooperation of other SUs on the performance of the secondary spectrum access in the cognitive radio networks. The results provided in this paper suggest that it is inevitable to take advantage of MIMO systems and cooperation of other 


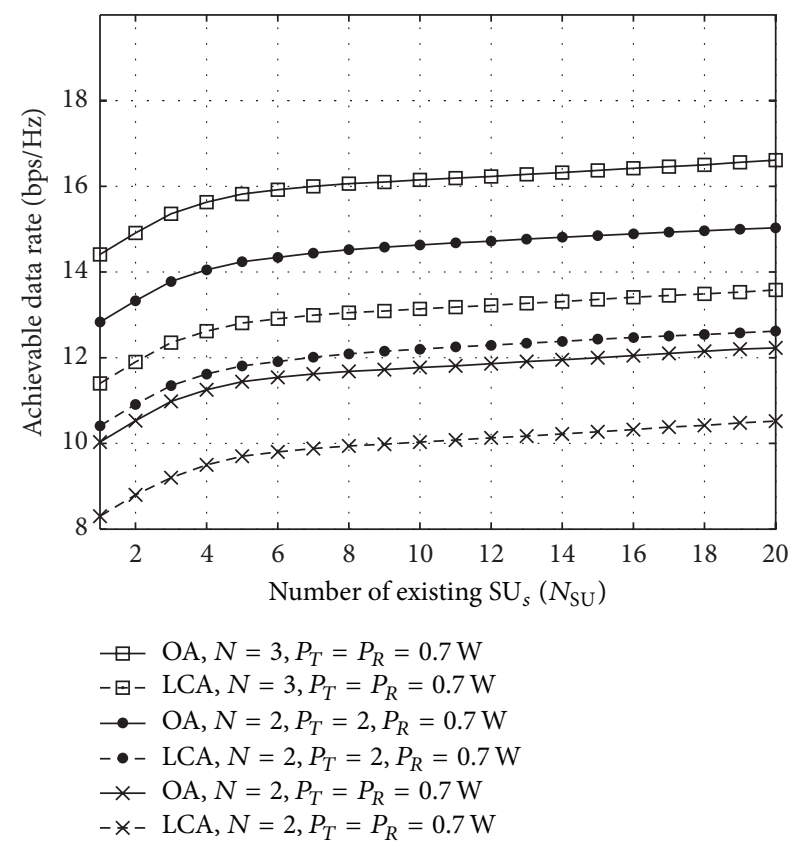

FIGURE 4: Achievable data rate in the desired link versus the number of existing SUs.

SUs, for the aim of opportunistic and dynamic spectrum access and to achieve larger data rates without inducing intolerable interference on the PUs.

\section{Conclusions}

In this work, an adaptive transmission strategy for underlay MIMO cooperative cognitive radio networks was proposed. It is assumed that when the PUs are present, the direct transmission by the SUs introduces intolerable interference on PUs. As a remedy and to maintain the performance quality of the SUs, the cooperation of one of the existing SUs was proposed not only to reduce the imposed interference on PUs but also to maximize the data rates in the desired SU link. Afterwards, the optimal solution of the joint problems of power allocation (both in the SU TX and the cooperating $\mathrm{SU})$ and relay selection was presented. However, due to high complexity of the optimal approach, a suboptimal approach with less complexity was further proposed. Meanwhile, the expected degradation in the system performance due to suboptimal approach was proved to be negligible, using simulations. Finally, an outage probability analysis was provided to examine the performance of the proposed MIMO cooperative cognitive radio network.

\section{Appendix}

Proof of Proposition 2. Our convention is that all eigenvalues are arranged in descending order. It was shown in [32] that if the SU TX works in spatial multiplexing mode, that is, the
SU TX transmits independent data streams from different antennas, the amplification matrix of $\mathrm{SU} i$ can be written as

$$
\mathbf{A}_{i}=\mathbf{V}_{r d, i} \Lambda_{\mathbf{A}_{i}} \mathbf{U}_{s r, i}^{H},
$$

where $\Lambda_{\mathbf{A}_{i}}$ is a diagonal matrix. Therefore, $\mathbf{A}_{i}$ can be considered as a matched filter along the singular vectors of the channel matrices. In order to use the results of [32] for the case of nonwhite transmit data of the SU TX and equivalently the transmit covariance matrix which is any arbitrary matrix $\mathbf{Q}_{i}$, we define the equivalent channel matrix $\widetilde{\mathbf{H}}_{s r, i}=\mathbf{H}_{s r, i} \mathbf{Q}_{i}^{-1 / 2}$. Hence, by adopting the same method, as in [32], for any given pair of $\mathbf{A}_{i}$ and $\widetilde{\mathbf{Q}}_{i}$, there always exists another pair $\mathbf{A}_{i, \text { opt }}$ and $\widetilde{\mathbf{Q}}_{i}$ that achieves better or equal data rate in the desired link. In this case, for the case of known $\mathbf{Q}_{i}$, (A.1) must be modified as

$$
\mathbf{A}_{i, \text { opt }}=\mathbf{V}_{r d, i} \Lambda_{\mathbf{A}_{i}} \widetilde{\mathbf{U}}_{s r, i}^{H} \text {, }
$$

where $\widetilde{\mathbf{U}}_{s r, i}$ is obtained by eigenvalue decomposition of $\widetilde{\mathbf{H}}_{s r, i} \widetilde{\mathbf{H}}_{s r, i}^{H} ;$ that is, $\widetilde{\mathbf{H}}_{s r, i} \widetilde{\mathbf{H}}_{s r, i}^{H}=\mathbf{H}_{s r, i} \widetilde{\mathbf{Q}}_{i} \mathbf{H}_{s r, i}^{H}=\widetilde{\mathbf{U}}_{s r, i} \widetilde{\Sigma}_{s r, i} \widetilde{\mathbf{U}}_{s r, i}^{H}$.

\section{Conflict of Interests}

The authors declare that there is no conflict of interests regarding the publication of this paper.

\section{References}

[1] S. Haykin, "Cognitive radio: brain-empowered wireless communications," IEEE Journal on Selected Areas in Communications, vol. 23, no. 2, pp. 201-220, 2005.

[2] Q. Zhao and B. M. Sadler, "A survey of dynamic spectrum access," IEEE Signal Processing Magazine, vol. 24, no. 3, pp. 7989, 2007.

[3] Y. Yu, W. Wang, C. Wang, F. Yan, and Y. Zhang, "Joint relay selection and power allocation with QoS support for cognitive radio networks," in Proceedings of the IEEE Wireless Communications and Networking Conference (WCNC '13), pp. 4516-4521, Shanghai, China, April 2013.

[4] K. J. Kim, T. Q. Duong, and X. Tran, "Performance analysis of cognitive spectrum-sharing single-carrier systems with relay selection," IEEE Transactions on Signal Processing, vol. 60, no. 12, pp. 6435-6449, 2012.

[5] M. G. Adian and H. Aghaeinia, "Optimal resource allocation for opportunistic spectrum access in multiple-input multiple-output-orthogonal frequency division multiplexing based cooperative cognitive radio networks," IET Signal Processing, vol. 7, no. 7, pp. 549-557, 2013.

[6] P. Ubaidulla and S. Aïssa, "Optimal relay selection and power allocation for cognitive two-way relaying networks," IEEE Wireless Communications Letters, vol. 1, no. 3, pp. 225-228, 2012.

[7] L. Li, X. Zhou, H. Xu, G. Y. Li, D. Wang, and A. Soong, "Simplified relay selection and power allocation in cooperative cognitive radio systems," IEEE Transactions on Wireless Communications, vol. 10, no. 1, pp. 33-36, 2011.

[8] P. Li, S. Guo, W. Zhuang, and B. Ye, "On efficient resource allocation for cognitive and cooperative communications," IEEE Journal on Selected Areas in Communications, vol. 32, no. 2, pp. 264-273, 2014. 
[9] M. G. Adian, H. Aghaeinia, and Y. Norouzi, "pectrum sharing and power allocation in multi-input-multi-output multi-band underlay cognitive radio networks," IET Communications, vol. 7, no. 11, pp. 1140-1150, 2013.

[10] M. G. Adian and H. Aghaeinia, "An auction-based approach for spectrum leasing in cooperative cognitive radio networks: when to lease and how much to be leased," Wireless Networks, vol. 20, pp. 411-422, 2014.

[11] M. G. Adian and H. Aghaeinia, "Spectrum sharing and power allocation in multiple-in multiple-out cognitive radio networks via pricing," IET Communications, vol. 6, no. 16, pp. 2621-2629, 2012.

[12] M. G. Adian and H. Aghaeinia, "Resource allocation in MIMOOFDM based cooperative cognitive radio networks," IEEE Transactions on Communications, vol. 62, no. 7, pp. 2224-2235, 2014.

[13] M. G. Adian and H. Aghaeinia, "Low complexity resource allocation in MIMO-OFDM-based cooperative cognitive radio networks," Transactions on Emerging Telecommunications Technology, 2014.

[14] M. G. Adian, "Beamforming with reduced complexity in MIMO cooperative cognitive radio networks," Journal of Optimization, vol. 2014, Article ID 325217, 10 pages, 2014.

[15] M. G. Adian and H. Aghaeinia, "Optimal resource allocation in heterogeneous MIMO cognitive radio networks," Wireless Personal Communication, vol. 76, no. 1, pp. 23-39, 2014.

[16] M. G. Adian and H. Aghaeinia, "A two-level cooperative gamebased approach for joint relay selection and distributed resource allocation in MIMO-OFDM-based cooperative cognitive radio networks," Transactions on Emerging Telecommunications Technology, 2014.

[17] J. Liu, N. B. Shroff, and H. D. Sherali, "Optimal power allocation in multi-relay MIMO cooperative networks: theory and algorithms," IEEE Journal on Selected Areas in Communications, vol. 30, no. 2, pp. 331-340, 2012.

[18] C. Stevenson, G. Chouinard, Z. Lei, W. Hu, S. Shellhammer, and W. Caldwell, "IEEE 802.22: the first cognitive radio wireless regional area network standard," IEEE Communications Magazine, vol. 47, no. 1, pp. 130-138, 2009.

[19] J. Jia, J. Zhang, and Q. Zhang, "Cooperative relay for cognitive radio networks," in Proceedings of the 28th Conference on Computer Communications (INFOCOM '09), pp. 2304-2312, IEEE, Rio de Janeiro, Brazil, April 2009.

[20] V. Brik, E. Rozner, S. Banerjee, and P. Bahl, "DSAP: a protocol for coordinated spectrum access," in Proceedings of the 1st IEEE International Symposium on New Frontiers in Dynamic Spectrum Access Networks (DySPAN '05), pp. 611-614, November 2005.

[21] G. Zhao, C. Yang, G. Y. Li, D. Li, and A. C. K. Soong, "Power and channel allocation for cooperative relay in cognitive radio networks," IEEE Journal on Selected Topics in Signal Processing, vol. 5, no. 1, pp. 151-159, 2011.

[22] G. Bansal, M. J. Hossain, and V. K. Bhargava, "Optimal and suboptimal power allocation schemes for OFDM-based cognitive radio systems," IEEE Transactions on Wireless Communications, vol. 7, no. 11, pp. 4710-4718, 2008.

[23] Y. Ma, D. I. Kim, and Z. Wu, "Optimization of OFDMAbased cellular cognitive radio networks," IEEE Transactions on Communications, vol. 58, no. 8, pp. 2265-2276, 2010.

[24] X. Kang, H. Garg, Y. Liang, and R. Zhang, "Optimal power allocation for OFDM-based cognitive radio with new primary transmission protection criteria," IEEE Transactions on Wireless Communications, vol. 9, no. 6, pp. 2066-2075, 2010.

[25] L. Zhang, Y.-C. Liang, and Y. Xin, "Joint beamforming and power allocation for multiple access channels in cognitive radio networks," IEEE Journal on Selected Areas in Communications, vol. 26, no. 1, pp. 38-51, 2008.

[26] L. Zhang, Y. Xin, Y. Liang, and H. V. Poor, “Cognitive multiple access channels: optimal power allocation for weighted sum rate maximization," IEEE Transactions on Communications, vol. 57, no. 9, pp. 2754-2762, 2009.

[27] S. Boyd and L. Vandenberghe, Convex Optimization, Cambridge University Press, 2004.

[28] W. Yu and R. Lui, "Dual methods for nonconvex spectrum optimization of multicarrier systems," IEEE Transactions on Communications, vol. 54, no. 7, pp. 1310-1322, 2006.

[29] E. Telatar, "Capacity of multi-antenna Gaussian channels," European Transactions on Telecommunications, vol. 10, no. 6, pp. 585-598, 1999.

[30] E. Biglieri, G. Caire, and G. Taricco, "Limiting performance of block-fading channels with multiple antennas," IEEE Transactions on Information Theory, vol. 47, no. 4, pp. 1273-1289, 2001.

[31] B. M. Hochwald, T. L. Marzetta, and V. Tarokh, "Multipleantenna channel hardening and its implications for rate feedback and scheduling," IEEE Transactions on Information Theory, vol. 50, no. 9, pp. 1893-1909, 2004.

[32] X. Tang and Y. Hua, "Optimal design of non-regenerative MIMO wireless relays," IEEE Transactions on Wireless Communications, vol. 6, no. 4, pp. 1398-1407, 2007. 


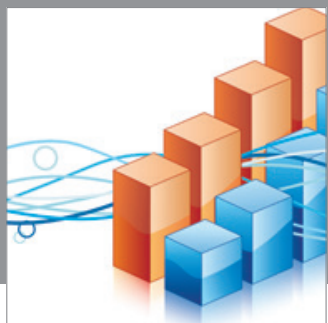

Advances in

Operations Research

mansans

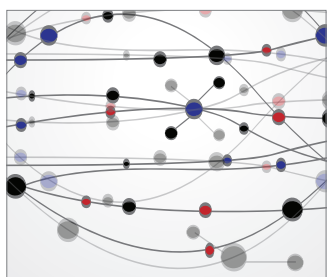

The Scientific World Journal
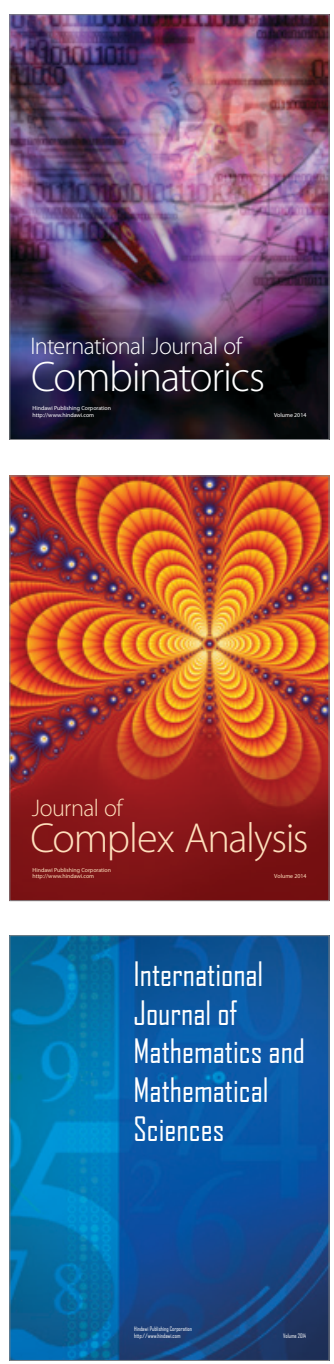
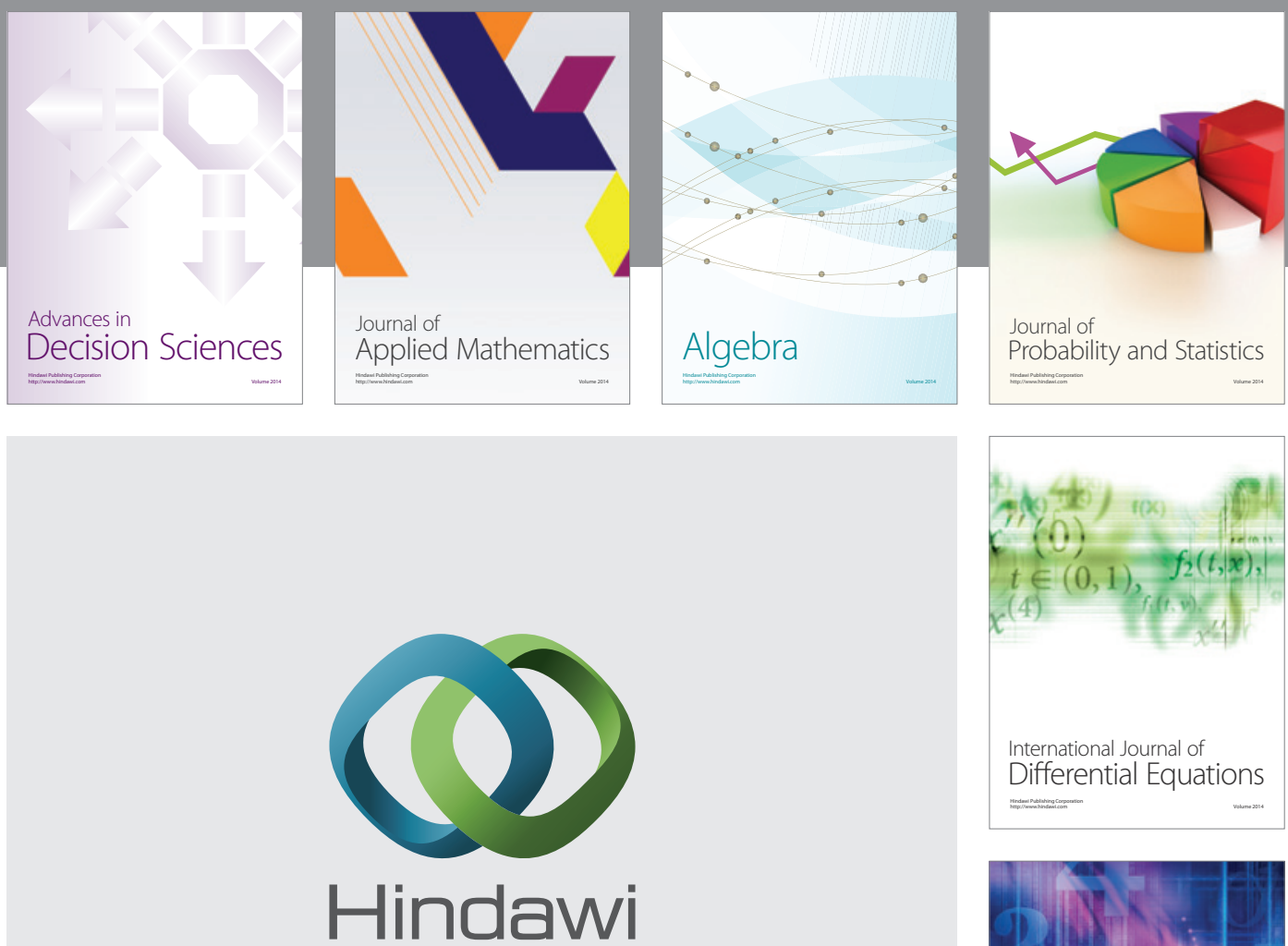

Submit your manuscripts at http://www.hindawi.com
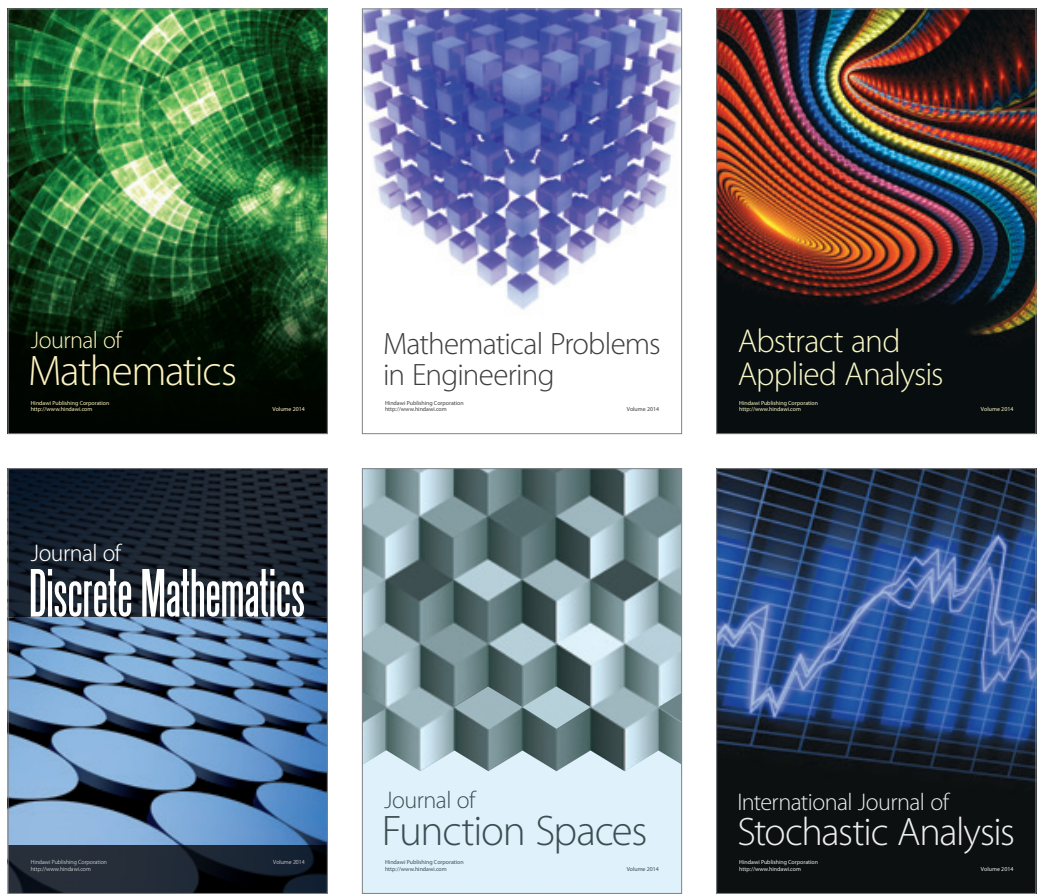

Journal of

Function Spaces

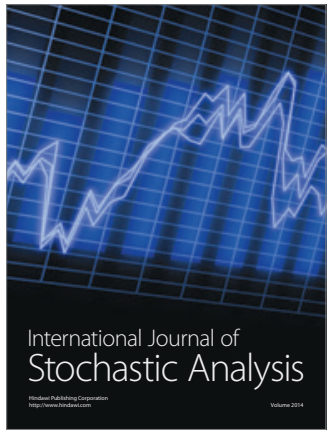

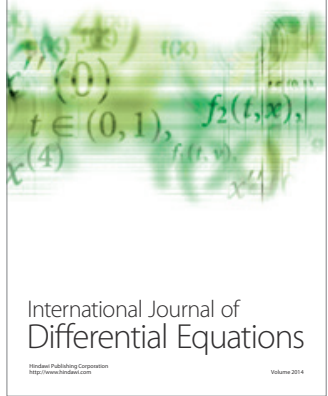
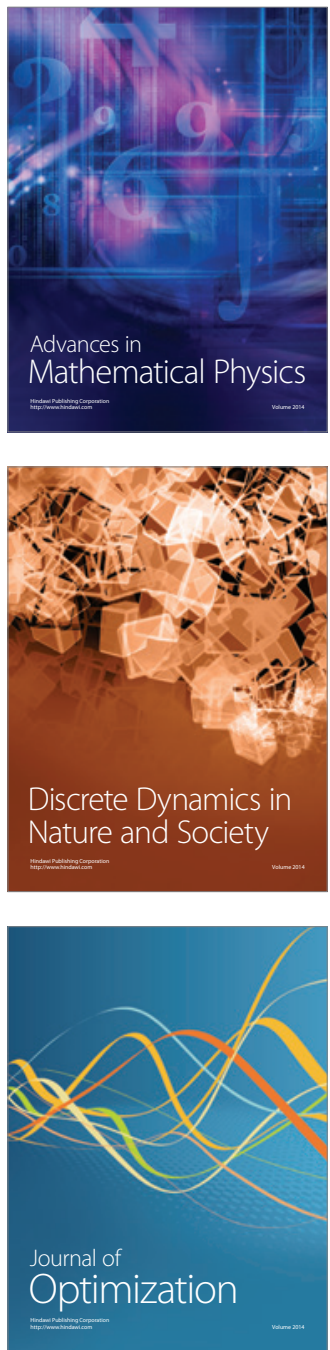